\title{
TITLE:
}

\section{About the Connes embedding conjecture}

$\operatorname{AUTHOR}(\mathrm{S})$ :

Ozawa, Narutaka

\section{CITATION:}

Ozawa, Narutaka. About the Connes embedding conjecture. Japanese Journal of Mathematics 2013, 8(1): 147-183

ISSUE DATE:

2013-03

URL:

http://hdl.handle.net/2433/173118

\section{RIGHT:}

The final publication is available at www.springerlink.com; This is not the published version. Please cite only the published version.; この論文 は出版社版でありません。引用の際には出版社版をご確認ご利用くだ さい。 


\title{
ABOUT THE CONNES EMBEDDING CONJECTURE - algebraic approaches-
}

\author{
NARUTAKA OZAWA
}

\begin{abstract}
In his celebrated paper in 1976, A. Connes casually remarked that any finite von Neumann algebra ought to be embedded into an ultraproduct of matrix algebras, which is now known as the Connes embedding conjecture or problem. This conjecture became one of the central open problems in the field of operator algebras since E. Kirchberg's seminal work in 1993 that proves it is equivalent to a variety of other seemingly totally unrelated but important conjectures in the field. Since then, many more equivalents of the conjecture have been found, also in some other branches of mathematics such as noncommutative real algebraic geometry and quantum information theory. In this note, we present a survey of this conjecture with a focus on the algebraic aspects of it.
\end{abstract}

1. Introduction. The Connes Embedding Conjecture $([\mathrm{Co}])$ is considered as one of the most important open problems in the field of operator algebras. It asserts that any finite von Neumann algebra is approximable by matrix algebras in a suitable sense. It turns out, most notably by Kirchberg's seminal work ([Ki1]), that the Connes Embedding Conjecture is equivalent to a variety of other important conjectures, which touches most of the subfields of operator algebras, and also some other branches of mathematics such as noncommutative real algebraic geometry ( $[\mathrm{Sm}])$ and quantum information theory. In this note, we look at the algebraic aspects of this conjecture. (See [BO, Ki1, Oz1] for the analytic aspects.) This leads to a study of the $\mathrm{C}^{*}$-algebraic aspect of noncommutative real algebraic geometry in terms of semi-pre- $\mathrm{C}^{*}$-algebras. Specifically, we treat some easy parts of Positivstellensätze of Putinar $([\mathrm{Pu}])$, Helton-McCullough $([\mathrm{HM}])$, and Schmüdgen-Bakonyi-Timotin $([\mathrm{BT}])$. We then treat their tracial analogue by KlepSchweighofer $([\mathrm{KS}])$, which is equivalent to the Connes Embedding Conjecture. We give new proofs of Kirchberg's theorems on the tensor product $\mathrm{C}^{*} \mathbb{F}_{d} \otimes \mathbb{B}\left(\ell_{2}\right)$ and on the equivalence between the Connes Embedding Conjecture and Kirchberg's Conjecture. We also look at Tsirelson's Problem in quantum information theory ([Fr, J+, Ts $]$ ), and prove it is

Date: April 4, 2013.

2010 Mathematics Subject Classification. 16W80; 46L89, 81P15.

Key words and phrases. Connes Embedding Conjecture, Kirchberg's Conjecture, Tsirelson's Problem, semi-pre-C*-algebras, noncommutative real algebraic geometry.

Partially supported by JSPS (23540233) and by the Danish National Research Foundation (DNRF) through the Centre for Symmetry and Deformation. 
again equivalent to the Connes Embedding Conjecture. This paper is an expanded lecture note for the author's lecture for "Masterclass on sofic groups and applications to operator algebras" (University of Copenhagen, 5-9 November 2012). The author gratefully acknowledges the kind hospitality provided by University of Copenhagen during his stay in Fall 2012. He also would like to thank Professor Andreas Thom for valuable comments on this note.

2. Ground assumption. We deal with unital $*$-algebras over $\mathbb{k} \in\{\mathbb{C}, \mathbb{R}\}$, and every algebra is assumed to be unital, unless it is clearly not so. The unit of an algebra is simply denoted by 1 and all homomorphisms and representations between algebras are assumed to preserve the units. We denote by in the imaginary unit, and by $\lambda^{*}$ the complex conjugate of $\lambda \in \mathbb{C}$. In case $\mathbb{k}=\mathbb{R}$, one has $\lambda^{*}=\lambda$ for all $\lambda \in \mathbb{k}$.

3. Semi-pre-C $\mathrm{C}^{*}$-algebras. We will give the definition and examples of semi-pre- $\mathrm{C}^{*}$-algebras. Recall that a unital algebra $\mathcal{A}$ is called a $*$-algebra if it is equipped with a map $x \mapsto x^{*}$ satisfying the following properties:

(i) $1^{*}=1$ and $\left(x^{*}\right)^{*}=x$ for every $x \in \mathcal{A}$;

(ii) $(x y)^{*}=y^{*} x^{*}$ for every $x, y \in \mathcal{A}$;

(iii) $(\lambda x+y)^{*}=\lambda^{*} x^{*}+y^{*}$ for every $x, y \in \mathcal{A}$ and $\lambda \in \mathbb{k}$.

The sets of hermitian elements and unitary (orthogonal) elements are written respectively as

$$
\mathcal{A}_{\mathrm{h}}:=\left\{a \in \mathcal{A}: a^{*}=a\right\} \text { and } \mathcal{A}_{\mathrm{u}}:=\left\{u \in \mathcal{A}: u^{*} u=1=u u^{*}\right\} .
$$

Every element $x \in \mathcal{A}$ decomposes uniquely as a sum $x=a+b$ of an hermitian element $a$ and a skew-hermitian element $b$. The set of hermitian elements is an $\mathbb{R}$-vector space. We say a linear map $\varphi$ between $*$-spaces is self-adjoint if $\varphi^{*}=\varphi$, where $\varphi^{*}$ is defined by $\varphi^{*}(x)=\varphi\left(x^{*}\right)^{*}$. We call a subset $\mathcal{A}_{+} \subset \mathcal{A}_{\mathrm{h}}$ a $*$-positive cone (commonly known as a quadratic module) if it satisfies the following:

(i) $\mathbb{R}_{\geq 0} 1 \subset \mathcal{A}_{+}$and $\lambda a+b \in \mathcal{A}_{+}$for every $a, b \in \mathcal{A}_{+}$and $\lambda \in \mathbb{R}_{\geq 0}$;

(ii) $x^{*} a x \in \mathcal{A}_{+}$for every $a \in \mathcal{A}_{+}$and $x \in \mathcal{A}$.

For $a, b \in \mathcal{A}_{\mathrm{h}}$, we write $a \leq b$ if $b-a \in \mathcal{A}_{+}$. We say a linear map $\varphi$ between spaces with positivity is positive if it sends positive elements to positive elements (and often it is also required self-adjoint), and a positive linear map $\varphi$ is faithful if $a \geq 0$ and $\varphi(a)=0$ implies $a=0$. Given a $*$-positive cone $\mathcal{A}_{+}$, we define the $*$-subalgebra of bounded elements by

$$
\mathcal{A}^{\text {bdd }}=\left\{x \in \mathcal{A}: \exists R>0 \text { such that } x^{*} x \leq R 1\right\} .
$$

This is indeed a $*$-subalgebra of $\mathcal{A}$. For example, if $x$ is bounded and $x^{*} x \leq R 1$, then $x^{*}$ is also bounded and $x x^{*} \leq R 1$, because

$$
0 \leq R^{-1}\left(R 1-x x^{*}\right)^{2}=R 1-2 x x^{*}+R^{-1} x\left(x^{*} x\right) x^{*} \leq R 1-x x^{*} .
$$

Thus, if $\mathcal{A}$ is generated (as a *-algebra) by $\mathcal{S}$, then $\mathcal{S} \subset \mathcal{A}^{\text {bdd }}$ implies $\mathcal{A}=\mathcal{A}^{\text {bdd }}$. 
Definition. A unital $*$-algebra $\mathcal{A}$ is called a semi-pre-C*-algebra if it comes together with a $*$-positive cone $\mathcal{A}_{+}$satisfying the Combes axiom (also called the archimedean property) that $\mathcal{A}=\mathcal{A}^{\text {bdd }}$.

Since $h \leq\left(1+h^{2}\right) / 2$ for $h \in \mathcal{A}_{\mathrm{h}}$, one has $\mathcal{A}_{\mathrm{h}}=\mathcal{A}_{+}-\mathcal{A}_{+}$for a semi-pre-C*-algebra. We define the ideal of infinitesimal elements by

$$
I(\mathcal{A})=\left\{x \in \mathcal{A}: x^{*} x \leq \varepsilon 1 \text { for all } \varepsilon>0\right\}
$$

and the archimedean closure of the $*$-positive cone $\mathcal{A}_{+}$(or any other cone) by

$$
\operatorname{arch}\left(\mathcal{A}_{+}\right)=\left\{a \in \mathcal{A}_{\mathrm{h}}: a+\varepsilon 1 \in \mathcal{A}_{+} \text {for all } \varepsilon>0\right\} .
$$

The cone $\mathcal{A}_{+}$is said to be archimedean closed if $\mathcal{A}_{+}=\operatorname{arch}\left(\mathcal{A}_{+}\right)$. A $\mathrm{C}^{*}$-algebra $A$ is of course a semi-pre- $\mathrm{C}^{*}$-algebra, with a zero infinitesimal ideal and an archimedean closed $*$-positive cone

$$
A_{+}=\left\{x^{*} x: x \in A\right\} .
$$

If $A \subset \mathbb{B}(H)$ (here $\mathbb{B}(H)$ denotes the $\mathrm{C}^{*}$-algebra of the bounded linear operators on a Hilbert space $H$ over $\mathbb{k}$ ), then one also has

$$
A_{+}=\left\{a \in A_{\mathrm{h}}:\langle a \xi, \xi\rangle \geq 0 \text { for all } \xi \in H\right\} .
$$

Note that the condition $a$ being hermitian can not be dropped when $\mathbb{k}=\mathbb{R}$. It will be shown (Theorem 1) that if $\mathcal{A}$ is a semi-pre-C $\mathrm{C}^{*}$-algebra, then $\mathcal{A} / I(\mathcal{A})$ is a pre-C $\mathrm{C}^{*}$-algebra with a $*$-positive cone $\operatorname{arch}\left(\mathcal{A}_{+}\right)$.

Definition. We define the universal $\mathrm{C}^{*}$-algebra of a semi-pre- $\mathrm{C}^{*}$-algebra $\mathcal{A}$ as the $\mathrm{C}^{*}$ algebra $\mathrm{C}_{\mathrm{u}}^{*}(\mathcal{A})$ together with a positive $*$-homomorphism $\iota: \mathcal{A} \rightarrow \mathrm{C}_{\mathrm{u}}^{*}(\mathcal{A})$ which satisfies the following properties: $\iota(\mathcal{A})$ is dense in $\mathrm{C}_{\mathrm{u}}^{*}(\mathcal{A})$ and every positive $*$-representation $\pi$ of $\mathcal{A}$ on a Hilbert space $H$ extends to a $*$-representation $\bar{\pi}: \mathrm{C}_{\mathrm{u}}^{*}(\mathcal{A}) \rightarrow \mathbb{B}(H)$, i.e., $\pi=\bar{\pi} \circ \iota$. In other words, $\mathrm{C}_{\mathrm{u}}^{*}(\mathcal{A})$ is the separation and completion of $\mathcal{A}$ under the $\mathrm{C}^{*}$-semi-norm

$$
\sup \left\{\|\pi(a)\|_{\mathbb{B}(H)}: \pi \text { a positive } * \text {-representation on a Hilbert space } H\right\} \text {. }
$$

(We may restrict the dimension of $H$ by the cardinality of $\mathcal{A}$.)

We emphasize that only positive *-representations are considered. Every positive *homomorphism between semi-pre- $\mathrm{C}^{*}$-algebras extends to a positive $*$-homomorphism between their universal $\mathrm{C}^{*}$-algebras. It may happen that $\mathcal{A}_{+}=\mathcal{A}_{\mathrm{h}}$ and $\mathrm{C}_{\mathrm{u}}^{*}(\mathcal{A})=\{0\}$, which is still considered as a unital (?) $\mathrm{C}^{*}$-algebra. Every $*$-homomorphism between $\mathrm{C}^{*}$-algebras is automatically positive, has a norm-closed range, and maps the positive cone onto the positive cone of the range. However, this is not at all the case for semi-pre-C $\mathrm{C}^{*}$-algebras, as we will exhibit a prototypical example in Example 1. On the other hand, we note that if $\mathcal{A}$ is a norm-dense *-subalgebra of a $\mathrm{C}^{*}$-algebra $A$ such that $\operatorname{arch}\left(\mathcal{A}_{+}\right)=\mathcal{A} \cap A_{+}$, then every positive $*$-representation of $\mathcal{A}$ extends to a $*$-representation of $A$, i.e., $A=\mathrm{C}_{\mathrm{u}}^{*}(\mathcal{A})$. (Indeed, if $x \in \mathcal{A}$ has $\|x\|_{A}<1$, then $1-x^{*} x \in \mathcal{A}_{+}$and hence $\|\pi(x)\|<1$ for any positive 
*-representation $\pi$ of $\mathcal{A}$.) It should be easy to see that the following examples satisfy the axiom of semi-pre- $\mathrm{C}^{*}$-algebras.

Example 1. Let $\Gamma$ be a discrete group and $\mathbb{k}[\Gamma]$ be its group algebra over $\mathbb{k}$ :

$$
(f * g)(s)=\sum_{t \in \Gamma} f\left(s t^{-1}\right) g(t) \text { and } f^{*}(s)=f\left(s^{-1}\right)^{*} \text { for } f, g \in \mathbb{k}[\Gamma] .
$$

The canonical $*$-positive cone of $\mathbb{k}[\Gamma]$ is defined as the sums of hermitian squares,

$$
\mathbb{k}[\Gamma]_{+}=\left\{\sum_{i=1}^{n} \xi_{i}^{*} * \xi_{i}: n \in \mathbb{N}, \xi_{i} \in \mathbb{k}[\Gamma]\right\} .
$$

Then, $\mathbb{k}[\Gamma]$ is a semi-pre-C $\mathrm{C}^{*}$-algebra such that $\mathrm{C}_{\mathrm{u}}^{*}(\mathbb{k}[\Gamma])=\mathrm{C}^{*} \Gamma$, the full group $\mathrm{C}^{*}$-algebra of $\Gamma$, which is the universal $\mathrm{C}^{*}$-algebra generated by the unitary representations of $\Gamma$. There is another group $\mathrm{C}^{*}$-algebra. Recall that the left regular representation $\lambda$ of $\Gamma$ on $\ell_{2} \Gamma$ is defined by $\lambda(s) \delta_{t}=\delta_{s t}$ for $s, t \in \Gamma$, or equivalently by $\lambda(f) \xi=f * \xi$ for $f \in \mathbb{k}[\Gamma]$ and $\xi \in \ell_{2} \Gamma$. The reduced group $\mathrm{C}^{*}$-algebra $\mathrm{C}_{\mathrm{r}}^{*} \Gamma$ of $\Gamma$ is the $\mathrm{C}^{*}$-algebra obtained as the normclosure of $\lambda(\mathbb{k}[\Gamma])$ in $\mathbb{B}\left(\ell_{2} \Gamma\right)$. The group algebra $\mathbb{k}[\Gamma]$ is equipped with the corresponding $*$-positive cone

$$
\begin{aligned}
\mathbb{k}_{\mathrm{r}}[\Gamma]_{+} & =\left\{f \in \mathbb{k}[\Gamma]: \exists f_{n} \in \mathbb{k}[\Gamma]_{+} \text {such that } f_{n} \rightarrow f \text { pointwise }\right\} \\
& =\{f \in \mathbb{k}[\Gamma]: f \text { is of positive type }\}
\end{aligned}
$$

and the resultant semi-pre-C $\mathrm{C}^{*}$-algebra $\mathbb{k}_{\mathrm{r}}[\Gamma]$ satisfies $\mathrm{C}_{\mathrm{u}}^{*}\left(\mathbb{k}_{\mathrm{r}}[\Gamma]\right)=\mathrm{C}_{\mathrm{r}}^{*} \Gamma$. Indeed, if $f \in$ $\mathbb{k}[\Gamma] \cap \lambda^{-1}\left(\mathrm{C}_{\mathrm{r}}^{*} \Gamma_{+}\right)$, then for $\xi=\lambda(f)^{1 / 2} \delta_{1} \in \ell_{2} \Gamma$, one has $f=\xi^{*} * \xi$ and $f$ is the pointwise limit of $\xi_{n}^{*} * \xi_{n} \in \mathbb{k}[\Gamma]_{+}$, where $\xi_{n} \in \mathbb{k}[\Gamma]$ are such that $\left\|\xi_{n}-\xi\right\|_{2} \rightarrow 0$. On the other hand, if $f$ is of positive type (i.e., the kernel $(x, y) \mapsto f\left(x^{-1} y\right)$ is positive semidefinite), then $f=f^{*}$ and $\langle\lambda(f) \eta, \eta\rangle \geq 0$ for every $\eta \in \ell_{2} \Gamma$, which implies $\lambda(f) \in \mathrm{C}_{\mathrm{r}}^{*} \Gamma_{+}$. It follows that $\mathbb{k}_{\mathrm{r}}[\Gamma]_{+}=\operatorname{arch}\left(\mathbb{k}[\Gamma]_{+}\right)$if and only if $\Gamma$ is amenable (see Theorem 1 ).

Example 2. The $*$-algebra $\mathbb{k}\left[x_{1}, \ldots, x_{d}\right]$ of polynomials in $d$ commuting hermitian variables $x_{1}, \ldots, x_{d}$ is a semi-pre- $\mathrm{C}^{*}$-algebra, equipped with the $*$-positive cone

$$
\mathbb{k}\left[x_{1}, \ldots, x_{d}\right]_{+}=* \text {-positive cone generated by }\left\{1-x_{i}^{2}: i=1, \ldots, d\right\} .
$$

One has $\mathrm{C}_{\mathrm{u}}^{*}\left(\mathbb{k}\left[x_{1}, \ldots, x_{d}\right]\right)=C\left([-1,1]^{d}\right)$, the algebra of the continuous functions on $[-1,1]^{d}$, and $x_{i}$ is identified with the $i$-th coordinate projection.

Example 3. The $*$-algebra $\mathbb{k}\left\langle x_{1}, \ldots, x_{d}\right\rangle$ of polynomials in $d$ non-commuting hermitian variables $x_{1}, \ldots, x_{d}$ is a semi-pre- $\mathrm{C}^{*}$-algebra, equipped with the $*$-positive cone

$$
\mathbb{k}\left\langle x_{1}, \ldots, x_{d}\right\rangle_{+}=* \text {-positive cone generated by }\left\{1-x_{i}^{2}: i=1, \ldots, d\right\} .
$$

One has $\mathrm{C}_{\mathrm{u}}^{*}\left(\mathbb{k}\left\langle x_{1}, \ldots, x_{d}\right\rangle\right)=C([-1,1]) * \cdots * C([-1,1])$, the unital full free product of $d$-copies of $C([-1,1])$. 
Example 4. Let $\mathcal{A}$ and $\mathcal{B}$ be semi-pre-C'-algebras. We denote by $\mathcal{A} \otimes \mathcal{B}$ the algebraic tensor product over $\mathbb{k}$. There are two standard ways to make $\mathcal{A} \otimes \mathcal{B}$ into a semi-pre- $\mathrm{C}^{*}$ algebra. The first one, called the maximal tensor product and denoted by $\mathcal{A} \otimes{ }^{\max } \mathcal{B}$, is $\mathcal{A} \otimes \mathcal{B}$ equipped with

$$
\left(\mathcal{A} \otimes{ }^{\max } \mathcal{B}\right)_{+}=* \text {-positive cone generated by }\left\{a \otimes b: a \in \mathcal{A}_{+}, b \in \mathcal{B}_{+}\right\} .
$$

The second one, called the minimal tensor product and denoted by $\mathcal{A} \otimes{ }^{\min } \mathcal{B}$, is $\mathcal{A} \otimes \mathcal{B}$ equipped with

$$
\left(\mathcal{A} \otimes{ }^{\min } \mathcal{B}\right)_{+}=(\mathcal{A} \otimes \mathcal{B})_{\mathrm{h}} \cap\left(\iota_{A} \otimes \iota_{B}\right)^{-1}\left(\left(\mathrm{C}_{\mathrm{u}}^{*}(\mathcal{A}) \otimes_{\min } \mathrm{C}_{\mathrm{u}}^{*}(\mathcal{B})\right)_{+}\right) .
$$

(See Theorem 14 for a "better" description.) One has $\mathrm{C}_{\mathrm{u}}^{*}\left(\mathcal{A} \otimes^{\alpha} \mathcal{B}\right)=\mathrm{C}_{\mathrm{u}}^{*}(\mathcal{A}) \otimes_{\alpha} \mathrm{C}_{\mathrm{u}}^{*}(\mathcal{B})$ for $\alpha \in\{\max , \min \}$. The right hand side is the $\mathrm{C}^{*}$-algebra maximal (resp. minimal) tensor product (see $[\mathrm{BO}, \mathrm{Pi} 1]$ ). For $\mathcal{A}_{1} \subset \mathcal{A}_{2}$ and $\mathcal{B}_{1} \subset \mathcal{B}_{2}$, one has

$$
\left(\mathcal{A}_{1} \otimes^{\min } \mathcal{B}_{1}\right)_{+}=\left(\mathcal{A}_{1} \otimes^{\min } \mathcal{B}_{1}\right) \cap\left(\mathcal{A}_{2} \otimes^{\min } \mathcal{B}_{2}\right)_{+},
$$

but the similar identity need not hold for the maximal tensor product.

Example 5. The unital algebraic free product $\mathcal{A} * \mathcal{B}$ of semi-pre-C*-algebras $\mathcal{A}$ and $\mathcal{B}$, equipped with

$$
(\mathcal{A} * \mathcal{B})_{+}=* \text {-positive cone generated by }\left(\mathcal{A}_{+} \cup \mathcal{B}_{+}\right),
$$

is a semi-pre- $\mathrm{C}^{*}$-algebra, and $\mathrm{C}_{\mathrm{u}}^{*}(\mathcal{A} * \mathcal{B})=\mathrm{C}_{\mathrm{u}}^{*}(\mathcal{A}) * \mathrm{C}_{\mathrm{u}}^{*}(\mathcal{B})$, the unital full free product of the $\mathrm{C}^{*}$-algebras $\mathrm{C}_{\mathrm{u}}^{*}(\mathcal{A})$ and $\mathrm{C}_{\mathrm{u}}^{*}(\mathcal{B})$.

The following is very basic (cf. [Ci] and Proposition 15 in [Sm]).

Theorem 1. Let $\mathcal{A}$ be a semi-pre- $\mathrm{C}^{*}$-algebra and $\iota: \mathcal{A} \rightarrow \mathrm{C}_{\mathrm{u}}^{*}(\mathcal{A})$ be the universal $\mathrm{C}^{*}$-algebra of $\mathcal{A}$. Then, one has the following.

- $\operatorname{ker} \iota=I(\mathcal{A})$, the ideal of the infinitesimal elements.

- $\mathcal{A}_{\mathrm{h}} \cap \iota^{-1}\left(\mathrm{C}_{\mathrm{u}}^{*}(\mathcal{A})_{+}\right)=\operatorname{arch}\left(\mathcal{A}_{+}\right)$, the archimedean closure of $\mathcal{A}_{+}$.

Although it follows from the above theorem, we give here a direct proof of the fact that $\operatorname{arch}\left(\mathcal{A}_{+}\right) \cap\left(-\operatorname{arch}\left(\mathcal{A}_{+}\right)\right) \subset I(\mathcal{A})$. Indeed, if $h^{2} \leq 1$ and $-\varepsilon 1<h<\varepsilon 1$ for $\varepsilon \in(0,1)$, then one has

$$
0 \leq(1+h)(\varepsilon-h)(1+h)=\varepsilon(1+h)^{2}-h-h(2+h) h \leq(4 \varepsilon+\varepsilon) 1-(2-\varepsilon) h^{2},
$$

which implies $h^{2}<5 \varepsilon 1$. We postpone the proof and give corollaries to this theorem.

4. Positivstellensätze. We give a few results which say if an element $a$ is positive in a certain class of representations, then it is positive for an obvious reason. Such results are referred to as "Positivstellensätze." Recall that a $\mathrm{C}^{*}$-algebra $A$ is said to be residually finite dimensional (RFD) if finite-dimensional *-representations separate the elements of $A$, i.e., $\pi(a) \geq 0$ for all finite-dimensional $*$-representations $\pi$ implies $a \geq 0$ in $A$. All abelian $\mathrm{C}^{*}$-algebras and full group $\mathrm{C}^{*}$-algebras of residually finite amenable groups are 
RFD. Moreover, it is a well-known result of Choi that the full group $\mathrm{C}^{*}$-algebra $\mathrm{C}^{*} \mathbb{F}_{d}$ of the free group $\mathbb{F}_{d}$ of rank $d$ is RFD (see Theorem 26). In fact, finite representations (i.e., the unitary representations $\pi$ such that $\pi\left(\mathbb{F}_{d}\right)$ is finite) separate the elements of $\mathrm{C}^{*} \mathbb{F}_{d}$ ([LS]). However, we note that the full group $\mathrm{C}^{*}$-algebra of a residually finite group need not be RFD ([Be1]). We also note that the unital full free products of RFD $\mathrm{C}^{*}$-algebras is again $\mathrm{RFD}([\mathrm{EL}])$. In particular, $\mathrm{C}_{\mathrm{u}}^{*}\left(\mathbb{R}_{k}\left\langle x_{1}, \ldots, x_{d}\right\rangle\right)$ is $\mathrm{RFD}$. The results mentioned here have been proven for complex $\mathrm{C}^{*}$-algebras, but they are equally valid for real cases. See Section 7. Theorem 1, when combined with residual finite dimensionality, immediately implies the following Positivstellensätze (cf. [Pu, HM]).

Corollary 2. The following are true.

- Let $f \in \mathbb{k}[\Gamma]_{\mathrm{h}}$. Then, $\pi(f) \geq 0$ for every unitary representation $\pi$ if and only if $f \in \operatorname{arch}\left(\mathbb{k}[\Gamma]_{+}\right)$.

- The full group $\mathrm{C}^{*}$-algebra $\mathrm{C}^{*} \Gamma$ of a group $\Gamma$ is $R F D$ if and only if the following statement holds. If $f \in \mathbb{k}[\Gamma]_{\mathrm{h}}$ is such that $\pi(f) \geq 0$ for every finite-dimensional unitary representation $\pi$, then $f \in \operatorname{arch}\left(\mathbb{k}[\Gamma]_{+}\right)$.

- Let $f \in \mathbb{k}\left[x_{1}, \ldots, x_{d}\right]_{\mathrm{h}}$. Then, $f\left(t_{1}, \ldots, t_{d}\right) \geq 0$ for all $\left(t_{1}, \ldots, t_{d}\right) \in[0,1]^{d}$ if and only if $f \in \operatorname{arch}\left(\mathbb{k}\left[x_{1}, \ldots, x_{d}\right]_{+}\right)$. (See Example 2.)

- Let $f \in \mathbb{k}\left\langle x_{1}, \ldots, x_{d}\right\rangle_{\mathrm{h}}$. Then, $f\left(X_{1}, \ldots, X_{d}\right) \geq 0$ for all contractive hermitian matrices $X_{1}, \ldots, X_{d}$ if and only if $f \in \operatorname{arch}\left(\mathbb{k}_{k}\left\langle x_{1}, \ldots, x_{d}\right\rangle_{+}\right)$. (See Example 3.)

In some cases, the $*$-positive cones are already archimedean closed. We will see later (Theorem 26) this phenomenon for the free group algebras $\mathbb{k}\left[\mathbb{F}_{d}\right]$.

5. Eidelheit-Kakutani Separation Theorem. The most basic tool in functional analysis is the Hahn-Banach theorem. In this note, we will need an algebraic form of it, the Eidelheit-Kakutani Separation Theorem. We recall the algebraic topology on an $\mathbb{R}$-vector space $V$. Let $C \subset V$ be a convex subset. An element $c \in C$ is called an algebraic interior point of $C$ if for every $v \in V$ there is $\varepsilon>0$ such that $c+\lambda v \in C$ for all $|\lambda|<\varepsilon$. The convex cone $C$ is said to be algebraically solid if the set $C^{\circ}$ of algebraic interior points of $C$ is non-empty. Notice that for every $c \in C^{\circ}$ and $x \in C$, one has $\lambda c+(1-\lambda) x \in C^{\circ}$ for every $\lambda \in(0,1]$. In particular, $C^{\circ \circ}=C^{\circ}$ for every convex subset $C$. We can equip $V$ with a locally convex topology, called the algebraic topology, by declaring that any convex set that coincides with its algebraic interior is open. Then, every linear functional on $V$ is continuous with respect to the algebraic topology. Now Hahn-Banach separation theorem reads as follows.

Theorem 3 (Eidelheit-Kakutani $([\mathrm{Ba}]))$. Let $V$ be an $\mathbb{R}$-vector space, $C$ an algebraically solid cone, and $v \in V \backslash C$. Then, there is a non-zero linear functional $\varphi: V \rightarrow \mathbb{R}$ such that

$$
\varphi(v) \leq \inf _{c \in C} \varphi(c)
$$

In particular, $\varphi(v)<\varphi(c)$ for any algebraic interior point $c \in C$. 
Notice that the Combes axiom $\mathcal{A}=\mathcal{A}^{\text {bdd }}$ is equivalent to that the unit 1 is an algebraic interior point of $\mathcal{A}_{+} \subset \mathcal{A}_{\mathrm{h}}$ and $\operatorname{arch}\left(\mathcal{A}_{+}\right)$is the algebraic closure of $\mathcal{A}_{+}$in $\mathcal{A}_{\mathrm{h}}$. (This is where the Combes axiom is needed and it can be dispensed when the cone $\mathcal{A}_{+}$is algebraically closed. See Section 3.4 in [Sm].) Let $\mathcal{A}$ be a semi-pre-C*-algebra. A unital *-subspace $\mathcal{S} \subset \mathcal{A}$ is called a semi-operator system. Here, a $*$-subspace is a subspace which is closed under the $*$-operation. Existence of 1 in $\mathcal{S}$ ensures that $\mathcal{S}_{+}=\mathcal{S} \cap \mathcal{A}_{+}$ has enough elements to span $\mathcal{S}_{\mathrm{h}}$. A linear functional $\varphi: \mathcal{S} \rightarrow \mathbb{k}$ is called a state if $\varphi$ is self-adjoint, positive, and $\varphi(1)=1$. Note that if $\mathbb{k}=\mathbb{C}$, then $\mathcal{S}$ is spanned by $\mathcal{S}_{+}$and every positive linear functional is automatically self-adjoint. However, this is not the case when $\mathbb{k}=\mathbb{R}$. In any case, every $\mathbb{R}$-linear functional $\varphi: \mathcal{S}_{\mathrm{h}} \rightarrow \mathbb{R}$ extends uniquely to a self-adjoint linear functional $\varphi: \mathcal{S} \rightarrow \mathbb{k}$. We write $S(\mathcal{S})$ for the set of states on $\mathcal{S}$.

Corollary 4. Let $\mathcal{A}$ be a semi-pre-C*-algebra.

- Let $W \subset \mathcal{A}$ be a $*$-subspace and $v \in \mathcal{A}_{\mathrm{h}} \backslash\left(\mathcal{A}_{+}+W_{\mathrm{h}}\right)$. Then, there is a state $\varphi$ on $\mathcal{A}$ such that $\varphi(W)=\{0\}$ and $\varphi(v) \leq 0$.

- (Krein's extension theorem) Let $\mathcal{S} \subset \mathcal{A}$ be a semi-operator system. Then every state on $\mathcal{S}$ extends to a state on $\mathcal{A}$.

Proof. Since $\mathcal{A}_{+}+W_{\mathrm{h}}$ is an algebraically solid cone in $\mathcal{A}_{\mathrm{h}}$, one may find a non-zero linear functional $\varphi$ on $\mathcal{A}_{\mathrm{h}}$ such that

$$
\varphi(v) \leq \inf \left\{\varphi(c): c \in \mathcal{A}_{+}+W_{\mathrm{h}}\right\}
$$

Since $\varphi$ is non-zero, $\varphi(1)>0$ and one may assume that $\varphi(1)=1$. Thus the self-adjoint extension of $\varphi$ on $\mathcal{A}$, still denoted by $\varphi$, is a state such that $\varphi(v) \leq 0$ and $\varphi\left(W_{\mathrm{h}}\right)=\{0\}$. Let $x \in W$. Then, for every $\lambda \in \mathbb{k}$, one has

$$
\lambda \varphi(x)+(\lambda \varphi(x))^{*}=\varphi\left((\lambda x)+(\lambda x)^{*}\right)=0 .
$$

This implies $\varphi(W)=\{0\}$ in either case $\mathbb{k} \in\{\mathbb{C}, \mathbb{R}\}$.

For the second assertion, let $\varphi \in S(\mathcal{S})$ be given and consider the cone

$$
C=\left\{x \in \mathcal{S}_{\mathrm{h}}: \varphi(x) \geq 0\right\}+\mathcal{A}_{+} .
$$

It is not too hard to see that $C$ is an algebraically solid cone in $\mathcal{A}_{\mathrm{h}}$ and $v \notin C$ for any $v \in \mathcal{S}_{\mathrm{h}}$ such that $\varphi(v)<0$. Hence, one may find a state $\bar{\varphi}$ on $\mathcal{A}$ such that $\bar{\varphi}(C) \subset \mathbb{R}_{\geq 0}$. In the same way as above, one has that $\bar{\varphi}$ is zero on $\operatorname{ker} \varphi$, which means $\left.\bar{\varphi}\right|_{\mathcal{S}}=\varphi$.

6. GNS construction. We recall the celebrated GNS construction (Gelfand-NaimarkSegal construction), which provides *-representations out of states. Let a semi-pre- $\mathrm{C}^{*}$ algebra $\mathcal{A}$ and a state $\varphi \in S(\mathcal{A})$ be given. Then, $\mathcal{A}$ is equipped with a semi-inner product $\langle y, x\rangle=\varphi\left(x^{*} y\right)$, and it gives rise to a Hilbert space, which will be denoted by $L^{2}(\mathcal{A}, \varphi)$. We denote by $\hat{x}$ the vector in $L^{2}(\mathcal{A}, \varphi)$ that corresponds to $x \in \mathcal{A}$. Thus, $\langle\hat{y}, \hat{x}\rangle=\varphi\left(x^{*} y\right)$ and $\|\hat{x}\|=\varphi\left(x^{*} x\right)^{1 / 2}$. The left multiplication $x \mapsto a x$ by an element $a \in \mathcal{A}$ extends to a bounded linear operator $\pi_{\varphi}(a)$ on $L^{2}(\mathcal{A}, \varphi)$ such that $\pi_{\varphi}(a) \hat{x}=\widehat{a x}$ for $a, x \in \mathcal{A}$. (Observe 
that $a^{*} a \leq R 1$ implies $\left\|\pi_{\varphi}(a)\right\|^{2} \leq R$.) It follows that $\pi_{\varphi}: \mathcal{A} \rightarrow \mathbb{B}\left(L^{2}(\mathcal{A}, \varphi)\right)$ is a positive *-representation of $\mathcal{A}$ such that $\left\langle\pi_{\varphi}(a) \hat{1}, \hat{1}\right\rangle=\varphi(a)$.

If $\pi: \mathcal{A} \rightarrow \mathbb{B}(H)$ is a positive $*$-representation having a unit cyclic vector $\xi$, then $\varphi(a)=\langle\pi(a) \xi, \xi\rangle$ is a state on $\mathcal{A}$ and $\pi(x) \xi \mapsto \hat{x}$ extends to a unitary isomorphism between $H$ and $L^{2}(\mathcal{A}, \varphi)$ which intertwines $\pi$ and $\pi_{\varphi}$. Since every positive $*$-representation decomposes into a direct sum of cyclic representations, one may obtain the universal $\mathrm{C}^{*}$-algebra $\mathrm{C}_{\mathrm{u}}^{*}(\mathcal{A})$ of $\mathcal{A}$ as the closure of the image under the positive *-representation

$$
\bigoplus_{\varphi \in S(\mathcal{A})} \pi_{\varphi}: \mathcal{A} \rightarrow \mathbb{B}\left(\bigoplus_{\varphi \in S(\mathcal{A})} L^{2}(\mathcal{A}, \varphi)\right)
$$

We also make an observation that $\left(\mathcal{A} \otimes^{\min } \mathcal{B}\right)_{+}$in Example 4 coincides with

$$
\left\{c \in(\mathcal{A} \otimes \mathcal{B})_{\mathrm{h}}:(\varphi \otimes \psi)\left(z^{*} c z\right) \geq 0 \text { for all } \varphi \in S(\mathcal{A}), \psi \in S(\mathcal{B}), z \in \mathcal{A} \otimes \mathcal{B}\right\} .
$$

7. Real versus Complex. We describe here the relation between real and complex semi-pre- $\mathrm{C}^{*}$-algebras. Because the majority of the researches on $\mathrm{C}^{*}$-algebras are carried out for complex $\mathrm{C}^{*}$-algebras, we look for a method of reducing real problems to complex problems. Suppose $\mathcal{A}_{\mathbb{R}}$ is a real semi-pre-C ${ }^{*}$-algebra. Then, the complexification of $\mathcal{A}_{\mathbb{R}}$ is the complex semi-pre-C $\mathrm{C}^{*}$-algebra $\mathcal{A}_{\mathbb{C}}=\mathcal{A}_{\mathbb{R}}+\dot{\mathrm{i}} \mathcal{A}_{\mathbb{R}}$. The $*$-algebra structure (over $\mathbb{C}$ ) of $\mathcal{A}_{\mathbb{C}}$ is defined in an obvious way, and $\left(\mathcal{A}_{\mathbb{C}}\right)_{+}$is defined to be the $*$-positive cone generated by $\left(\mathcal{A}_{\mathbb{R}}\right)_{+}$:

$$
\left(\mathcal{A}_{\mathbb{C}}\right)_{+}=\left\{\sum_{i=1}^{n} z_{i}^{*} a_{i} z_{i}: n \in \mathbb{N}, a_{i} \in\left(\mathcal{A}_{\mathbb{R}}\right)_{+}, z_{i} \in \mathcal{A}_{\mathbb{C}}\right\} .
$$

(This is a temporary definition, and the official one will be given later. See Lemma 11.) Note that $\mathcal{A}_{\mathbb{R}} \cap\left(\mathcal{A}_{\mathbb{C}}\right)_{+}=\left(\mathcal{A}_{\mathbb{R}}\right)_{+}$. The complexification $\mathcal{A}_{\mathbb{C}}$ has an involutive and conjugatelinear $*$-automorphism defined by $x+\dot{\mathrm{i}} y \mapsto x-\dot{\mathrm{i}} y, x, y \in \mathcal{A}_{\mathbb{R}}$. Every complex semi-pre$\mathrm{C}^{*}$-algebra with an involutive and conjugate-linear $*$-automorphism arises in this way.

Lemma 5. Let $\pi_{\mathbb{R}}: \mathcal{A}_{\mathbb{R}} \rightarrow \mathcal{B}_{\mathbb{R}}$ be a $*$-homomorphism between real semi-pre-C ${ }^{*}$-algebras (resp. $\varphi_{\mathbb{R}}: \mathcal{A}_{\mathbb{R}} \rightarrow \mathbb{R}$ be a self-adjoint linear functional). Then, the complexification $\pi_{\mathbb{C}}: \mathcal{A}_{\mathbb{C}} \rightarrow \mathcal{B}_{\mathbb{C}}$ (resp. $\varphi_{\mathbb{C}}: \mathcal{A}_{\mathbb{C}} \rightarrow \mathbb{C}$ ) is positive if and only if $\pi_{\mathbb{R}}$ (resp. $\varphi_{\mathbb{R}}$ ) is so.

Proof. We only prove that $\varphi_{\mathbb{C}}$ is positive if $\varphi_{\mathbb{R}}$ is so. The rest is trivial. Let $b=\sum_{i} z_{i}^{*} a_{i} z_{i} \in$ $\left(\mathcal{A}_{\mathbb{C}}\right)_{+}$be arbitrary, where $a_{i} \in\left(\mathcal{A}_{\mathbb{R}}\right)_{+}$and $z_{i}=x_{i}+\dot{\mathrm{i}} y_{i}$. Then, $b=\sum_{i}\left(x_{i}^{*} a_{i} x_{i}+y_{i}^{*} a_{i} y_{i}\right)+$ i $\sum_{i}\left(x_{i}^{*} a_{i} y_{i}-y_{i}^{*} a_{i} x_{i}\right)$. Since $x_{i}^{*} a_{i} y_{i}-y_{i}^{*} a_{i} x_{i}$ is skew-hermitian, one has $\varphi_{\mathbb{R}}\left(x_{i}^{*} a_{i} y_{i}-y_{i}^{*} a_{i} x_{i}\right)=$ 0 , and $\varphi_{\mathbb{C}}(b)=\varphi_{\mathbb{R}}\left(\sum_{i} x_{i}^{*} a_{i} x_{i}+y_{i}^{*} a_{i} y_{i}\right) \geq 0$. This shows $\varphi_{\mathbb{C}}$ is positive.

We note that if $H_{\mathbb{C}}$ denotes the complexification of a real Hilbert space $H_{\mathbb{R}}$, then $\mathbb{B}\left(H_{\mathbb{R}}\right)_{\mathbb{C}}=\mathbb{B}\left(H_{\mathbb{C}}\right)$. Thus every positive $*$-representation of a real semi-pre-C ${ }^{*}$-algebra $\mathcal{A}_{\mathbb{R}}$ on $H_{\mathbb{R}}$ extends to a positive $*$-representation of its complexification $\mathcal{A}_{\mathbb{C}}$ on $H_{\mathbb{C}}$. Conversely, if $\pi$ is a positive $*$-representation of $\mathcal{A}_{\mathbb{C}}$ on a complex Hilbert space $H_{\mathbb{C}}$, then its restriction to $\mathcal{A}_{\mathbb{R}}$ is a positive $*$-representation on the realification of $H_{\mathbb{C}}$. The realification of a 
complex Hilbert space $H_{\mathbb{C}}$ is the real Hilbert space $H_{\mathbb{C}}$ equipped with the real inner product $\langle\eta, \xi\rangle_{\mathbb{R}}=\Re\langle\eta, \xi\rangle$. Therefore, we arrive at the conclusion that $\mathrm{C}_{\mathrm{u}}^{*}\left(\mathcal{A}_{\mathbb{R}}\right)_{\mathbb{C}}=\mathrm{C}_{\mathrm{u}}^{*}\left(\mathcal{A}_{\mathbb{C}}\right)$. We also see that $(\mathbb{R}[\Gamma])_{\mathbb{C}}=\mathbb{C}[\Gamma],\left(\mathcal{A}_{\mathbb{R}} \otimes \mathcal{B}_{\mathbb{R}}\right)_{\mathbb{C}}=\mathcal{A}_{\mathbb{C}} \otimes \mathcal{B}_{\mathbb{C}},\left(\mathcal{A}_{\mathbb{R}} * \mathcal{B}_{\mathbb{R}}\right)_{\mathbb{C}}=\mathcal{A}_{\mathbb{C}} * \mathcal{B}_{\mathbb{C}}$, etc.

8. Proof of Theorem 1. We only prove the first assertion of Theorem 1. The proof of the second is very similar. We will prove a stronger assertion that

$$
\|\iota(x)\|_{\mathrm{C}_{\mathbf{u}}^{*}(\mathcal{A})}=\inf \left\{R>0: R^{2} 1-x^{*} x \in \mathcal{A}_{+}\right\} .
$$

The inequality $\leq$ trivially follows from the $\mathrm{C}^{*}$-identity. For the converse, assume that the right hand side is non-zero, and choose $\lambda>0$ such that $\lambda^{2} 1-x^{*} x \notin \mathcal{A}_{+}$. By Corollary 4 , there is $\varphi \in S(\mathcal{A})$ such that $\varphi\left(\lambda^{2} 1-x^{*} x\right) \leq 0$. Thus for the GNS representation $\pi_{\varphi}$, one has

$$
\left\|\pi_{\varphi}(x)\right\| \geq\left\|\pi_{\varphi}(x) \hat{1}\right\|=\varphi\left(x^{*} x\right)^{1 / 2} \geq \lambda .
$$

It follows that $\|\iota(x)\| \geq \lambda$.

9. Trace positive elements. Let $\mathcal{A}$ be a semi-pre-C*-algebra. A state $\tau$ on $\mathcal{A}$ is called a tracial state if $\tau(x y)=\tau(y x)$ for all $x, y \in \mathcal{A}$, or equivalently if $\tau$ is zero on the $*-$ subspace $K=\operatorname{span}\{x y-y x: x, y \in \mathcal{A}\}$ spanned by commutators in $\mathcal{A}$. We denote by $T(\mathcal{A})$ the set of tracial states on $\mathcal{A}$ (which may be empty). Associated with $\tau \in T(\mathcal{A})$ is a finite von Neumann algebra $\left(\pi_{\tau}(\mathcal{A})^{\prime \prime}, \tau\right)$, which is the von Neumann algebra generated by $\pi_{\tau}(\mathcal{A}) \subset \mathbb{B}\left(L^{2}(\mathcal{A}, \tau)\right)$ with the faithful normal tracial state $\tau(a)=\langle a \hat{1}, \hat{1}\rangle$ that extends the original $\tau$. Recall that a finite von Neumann algebra is a pair $(M, \tau)$ of a von Neumann algebra and a faithful normal tracial state $\tau$ on $M$. The following theorem is proved in $[\mathrm{KS}]$ for the algebra in Example 3 and in $[\mathrm{JP}]$ for the free group algebras, but the proof equally works in the general setting. We note that for some groups $\Gamma$, notably for $\Gamma=\mathrm{SL}_{3}(\mathbb{Z})([\mathrm{Be} 2])$, it is possible to describe all the tracial states on $\mathbb{k}[\Gamma]$.

Theorem $6([\mathrm{KS}])$. Let $\mathcal{A}$ be a semi-pre-C $\mathrm{C}^{*}$-algebra, and $a \in \mathcal{A}_{\mathrm{h}}$. Then, the following are equivalent.

(1) $\tau(a) \geq 0$ for all $\tau \in T(\mathcal{A})$.

(2) $\tau(\pi(a)) \geq 0$ for every finite von Neumann algebra $(M, \tau)$ and every positive $*$-homomorphism $\pi: \mathcal{A} \rightarrow M$.

(3) $a \in \operatorname{arch}\left(\mathcal{A}_{+}+K_{\mathrm{h}}\right)$, where $K_{\mathrm{h}}=K \cap \mathcal{A}_{\mathrm{h}}=\operatorname{span}\left\{x^{*} x-x x^{*}: x \in \mathcal{A}\right\}$.

Proof. The equivalence (1) $\Leftrightarrow(2)$ follows from the GNS construction. We only prove $(1) \Rightarrow(3)$, as the converse is trivial. Suppose $a+\varepsilon 1 \notin \mathcal{A}_{+}+K_{\mathrm{h}}$ for some $\varepsilon>0$. Then, by Corollary 4 , there is $\tau \in S(\mathcal{A})$ such that $\tau(K)=\{0\}$ (i.e., $\tau \in T(\mathcal{A})$ ) and $\tau(a) \leq-\varepsilon<0$. 
10. Connes Embedding Conjecture. The Connes Embedding Conjecture (CEC) asserts that any finite von Neumann algebra $(M, \tau)$ with separable predual is embeddable into the ultrapower $R^{\omega}$ of the hyperfinite $\mathrm{II}_{1}$-factor $R$ (over $\mathbb{k} \in\{\mathbb{C}, \mathbb{R}\}$ ). Here an embedding means an injective $*$-homomorphism which preserves the tracial state. We note that if $\tau$ is a tracial state on a semi-pre-C*-algebra $\mathcal{A}$ and $\theta: \mathcal{A} \rightarrow N$ is a $\tau$-preserving *-homomorphism into a finite von Neumann algebra $(N, \tau)$, then $\theta$ extends to a $\tau$-preserving $*$-isomorphism from $\pi_{\tau}(\mathcal{A})^{\prime \prime}$ onto the von Neumann subalgebra generated by $\theta(\mathcal{A})$ in $N$ (which coincides with the ultraweak closure of $\theta(\mathcal{A})$ ). Hence, $(M, \tau)$ satisfies CEC if there is an ultraweakly dense $*$-subalgebra $\mathcal{A} \subset M$ which has a $\tau$-preserving embedding into $R^{\omega}$. In particular, CEC is equivalent to that for every countably generated semipre-C ${ }^{*}$-algebra $\mathcal{A}$ and $\tau \in T(\mathcal{A})$, there is a $\tau$-preserving $*$-homomorphism from $\mathcal{A}$ into $R^{\omega}$. We will see that this is equivalent to the tracial analogue of Positivstellensätze in Corollary 2. We first state a few equivalent forms of CEC. We denote by tr the tracial state $\frac{1}{N} \operatorname{Tr}$ on $\mathbb{M}_{N}(\mathbb{k})$.

Theorem 7. For a finite von Neumann algebra $(M, \tau)$ with separable predual, the following are equivalent.

(1) $(M, \tau)$ satisfies $C E C$, i.e., $M \hookrightarrow R^{\omega}$.

(2) Let $d \in \mathbb{N}$ and $x_{1}, \ldots, x_{d} \in M$ be hermitian contractions. Then, for every $m \in \mathbb{N}$ and $\varepsilon>0$, there are $N \in \mathbb{N}$ and hermitian contractions $X_{1}, \ldots, X_{d} \in \mathbb{M}_{N}(\mathbb{k})$ such that

$$
\left|\tau\left(x_{i_{1}} \cdots x_{i_{k}}\right)-\operatorname{tr}\left(X_{i_{1}} \cdots X_{i_{k}}\right)\right|<\varepsilon
$$

for all $k \leq m$ and $i_{j} \in\{1, \ldots, d\}$.

(3) Assume $\mathbb{k}=\mathbb{C}$ (or replace $M$ with its complexification in case $\mathbb{k}=\mathbb{R}$ ). Let $d \in \mathbb{N}$ and $u_{1}, \ldots, u_{d} \in M$ be unitary elements. Then, for every $\varepsilon>0$, there are $N \in \mathbb{N}$ and unitary matrices $U_{1}, \ldots, U_{d} \in \mathbb{M}_{N}(\mathbb{C})$ such that

$$
\left|\tau\left(u_{i}^{*} u_{j}\right)-\operatorname{tr}\left(U_{i}^{*} U_{j}\right)\right|<\varepsilon
$$

for all $i, j \in\{1, \ldots, d\}$.

In particular, CEC holds true if and only if every $(M, \tau)$ satisfies condition (2) and/or (3).

The equivalence $(1) \Leftrightarrow(2)$ is a rather routine consequence of the ultraproduct construction. For the equivalence to (3), see Theorem 27. Note that the assumption $\mathbb{k}=\mathbb{C}$ in condition (3) is essential because the real analogue of it is actually true ([DJ]). Since any finite von Neumann algebra $M$ with separable predual is embeddable into a $\mathrm{II}_{1}$-factor which is generated by two hermitian elements (namely $(M * R) \bar{\otimes} R$ ), to prove CEC, it is enough to verify the conjecture (2) for every $(M, \tau)$ and $d=2$. We observe that a real finite von Neumann algebra $\left(M_{\mathbb{R}}, \tau_{\mathbb{R}}\right)$ is embeddable into $R_{\mathbb{R}}^{\omega}$ (i.e., it satisfies CEC) if and only if its complexification $\left(M_{\mathbb{C}}, \tau_{\mathbb{C}}\right)$ is embeddable into $R_{\mathbb{C}}^{\omega}$. The "only if" direction is trivial and the "if" direction follows from the real $*$-homomorphism $\mathbb{M}_{N}(\mathbb{C}) \hookrightarrow \mathbb{M}_{2 N}(\mathbb{R})$, 
$a+\dot{\mathrm{i}} b \mapsto\left[\begin{array}{cc}a & b \\ -b & a\end{array}\right]$. A complex finite von Neumann algebra $(M, \tau)$ need not be a complexification of a real von Neumann algebra, but $M \oplus M^{\text {op }}$ is (isomorphic to the complexification of the realification of $M$ ). Therefore, $M$ satisfies CEC if and only if its realification satisfies it.

For a finite von Neumann algebra $(M, \tau)$ and $d \in \mathbb{N}$, we denote by $\mathcal{H}_{d}(M)$ the set of those $f \in \mathbb{k}\left\langle x_{1}, \ldots, x_{d}\right\rangle_{\mathrm{h}}$ such that $\tau\left(f\left(X_{1}, \ldots, X_{d}\right)\right) \geq 0$ for all hermitian contractions $X_{1}, \ldots, X_{d} \in M$. Further, let

$$
\mathcal{H}_{d}=\bigcap_{M} \mathcal{H}_{d}(M) \text { and } \mathcal{H}_{d}^{\text {fin }}=\bigcap_{N} \mathcal{H}_{d}\left(\mathbb{M}_{N}(\mathbb{k})\right)=\mathcal{H}_{d}(R) .
$$

Notice that $\mathcal{H}_{d}=\operatorname{arch}\left(\mathbb{k}\left\langle x_{1}, \ldots, x_{d}\right\rangle_{+}+K_{\mathrm{h}}\right)$ (see Example 3 and Theorem 6 ).

Corollary $8([\mathrm{KS}])$. Let $\mathbb{k} \in\{\mathbb{C}, \mathbb{R}\}$. Then one has the following.

- Let $(M, \tau)$ be a finite von Neumann algebra with separable predual. Then, $M$ satisfies $C E C$ if and only if $\mathcal{H}_{d}^{\text {fin }} \subset \mathcal{H}_{d}(M)$ for all d.

- CEC holds true if and only if $\mathcal{H}_{d}^{\text {fin }}=\operatorname{arch}\left(\mathbb{k}\left\langle x_{1}, \ldots, x_{d}\right\rangle_{+}+K_{\mathrm{h}}\right)$ for all $/$ some $d \geq 2$.

Proof. It is easy to see that condition (2) in Theorem 7 implies $\mathcal{H}_{d}^{\text {fin }} \subset \mathcal{H}_{d}(M)$. Conversely, suppose condition (2) does not hold for some $d \in \mathbb{N}, x_{1}, \ldots, x_{d} \in M, m \in \mathbb{N}$, and $\varepsilon>0$. We introduce the multi-index notation. For $\mathbf{i}=\left(i_{1}, \ldots, i_{k}\right), i_{j} \in\{1, \ldots, d\}$ and $k \leq m$, we denote $x_{\mathbf{i}}=x_{i_{1}} \cdots x_{i_{k}}$. It may happen that $\mathbf{i}$ is the null string $\emptyset$ and $x_{\emptyset}=1$. Then,

$$
C=\operatorname{closure}\left\{\left(\operatorname{tr}\left(X_{\mathbf{i}}\right)\right)_{\mathbf{i}}: N \in \mathbb{N}, X_{1}, \ldots, X_{d} \in \mathbb{M}_{N}(\mathbb{k})_{\mathrm{h}},\left\|X_{i}\right\| \leq 1\right\}
$$

is a convex set (consider a direct sum of matrices). Hence by Theorem 3 , there are $\lambda \in \mathbb{R}$ and $\alpha_{\mathbf{i}} \in \mathbb{k}$ such that

$$
\Re \sum_{\mathbf{i}} \alpha_{\mathbf{i}} \tau\left(x_{\mathbf{i}}\right)<\lambda \leq \inf _{\gamma \in C} \Re \sum_{\mathbf{i}} \alpha_{\mathbf{i}} \gamma_{\mathbf{i}} .
$$

Replacing $\alpha_{\mathbf{i}}$ with $\left(\alpha_{\mathbf{i}}+\alpha_{\mathbf{i}^{*}}^{*}\right) / 2$ (here $\mathbf{i}^{*}$ is the reverse of $\mathbf{i}$ ), we may omit $\Re$ from the above inequality. Further, arranging $\alpha_{\emptyset}$, we may assume $\lambda=0$. Thus $f=\sum_{\mathbf{i}} \alpha_{\mathbf{i}} x_{\mathbf{i}}$ belongs to $\mathcal{H}_{d}^{\text {fin }}$, but not to $\mathcal{H}_{d}(M)$. This completes the proof of the first half. The second half follows from this and Theorem 6 .

An analogue to the above also holds for $\mathbb{C}\left[\mathbb{F}_{d}\right]$.

Corollary 9 ([JP]). Let $\mathbb{k}=\mathbb{C}$. The following holds.

- Let $(M, \tau)$ be a finite von Neumann algebra with separable predual. Then, $M$ satisfies $C E C$ if and only if the following holds true: If $d \in \mathbb{N}$ and $\alpha \in \mathbb{M}_{d}(\mathbb{C})_{\mathrm{h}}$ satisfies that $\operatorname{tr}\left(\sum \alpha_{i, j} U_{i}^{*} U_{j}\right) \geq 0$ for every $N \in \mathbb{N}$ and $U_{1}, \ldots, U_{d} \in \mathbb{M}_{N}(\mathbb{C})_{\mathrm{u}}$, then it satisfies $\tau\left(\sum \alpha_{i, j} u_{i}^{*} u_{j}\right) \geq 0$ for every unitary elements $u_{1}, \ldots, u_{d} \in M$.

- CEC holds true if and only if for every $d \in \mathbb{N}$ and $\alpha \in \mathbb{M}_{d}(\mathbb{C})_{\mathrm{h}}$ the following holds true: If $\operatorname{tr}\left(\sum \alpha_{i, j} U_{i}^{*} U_{j}\right) \geq 0$ for every $N \in \mathbb{N}$ and $U_{1}, \ldots, U_{d} \in \mathbb{M}_{N}(\mathbb{C})_{\mathrm{u}}$, then $\sum \alpha_{i, j} s_{i}^{*} s_{j} \in$ $\operatorname{arch}\left(\mathbb{C}\left[\mathbb{F}_{d}\right]_{+}+K_{\mathrm{h}}\right)$, where $s_{1}, \ldots, s_{d}$ are the free generators of $\mathbb{F}_{d}$. 
11. Matrix algebras over semi-pre-C*-algebras. We describe here how to make the $n \times n$ matrix algebra $\mathbb{M}_{n}(\mathcal{A})$ over a semi-pre- $\mathrm{C}^{*}$-algebra $\mathcal{A}$ into a semi-pre-C*-algebra. We note that $x^{*}=\left[x_{j, i}^{*}\right]_{i, j}$ for $x=\left[x_{i, j}\right]_{i, j} \in \mathbb{M}_{n}(\mathcal{A})$. We often identify $\mathbb{M}_{n}(\mathcal{A})$ with $\mathbb{M}_{n}(\mathbb{k}) \otimes \mathcal{A}$. There are two natural choices of the $*$-positive cone for $\mathbb{M}_{n}(\mathcal{A})$. The first and the official one is

$$
\begin{aligned}
\mathbb{M}_{n}(\mathcal{A})_{+} & =* \text {-positive cone generated by }\left\{\operatorname{diag}\left(a_{1}, \ldots, a_{n}\right): a_{i} \in \mathcal{A}_{+}\right\} \\
& =\left\{\left[\sum_{k=1}^{m} x_{k, i}^{*} a_{k} x_{k, j}\right]_{i, j}: m \in \mathbb{N}, a_{k} \in \mathcal{A}_{+},\left[x_{k, i}\right]_{k, i} \in \mathbb{M}_{m, n}(\mathcal{A})\right\}
\end{aligned}
$$

The second one is larger:

$$
\mathbb{M}_{n}(\mathcal{A})_{+}^{\prime}=\left\{\left[a_{i, j}\right] \in \mathbb{M}_{n}(\mathcal{A})_{\mathrm{h}}: \varphi\left(\sum_{i, j} x_{i}^{*} a_{i, j} x_{j}\right) \geq 0 \text { for all } x_{1}, \ldots, x_{n} \in \mathcal{A}, \varphi \in S(\mathcal{A})\right\} .
$$

(One may notice that the first one is $\mathbb{M}_{n}(\mathbb{k}) \otimes^{\max } \mathcal{A}$ and the second is $\mathbb{M}_{n}(\mathbb{k}) \otimes^{\min } \mathcal{A}$.) In case where $\mathcal{A}$ is a $\mathrm{C}^{*}$-algebra, these cones coincide and are archimedean closed. In either definition, one has $\mathrm{C}_{\mathrm{u}}^{*}\left(\mathbb{M}_{n}(\mathcal{A})\right)=\mathbb{M}_{n}\left(\mathrm{C}_{\mathrm{u}}^{*}(\mathcal{A})\right)$, because every $*$-representation of $\mathbb{M}_{n}(\mathcal{A})$ is of the form id $\otimes \pi: \mathbb{M}_{n}(\mathcal{A}) \rightarrow \mathbb{M}_{n}(\mathbb{B}(H)) \cong \mathbb{B}\left(\ell_{2}^{n} \otimes H\right)$. Therefore, by Theorem 1 , we arrive at the following conclusion.

Lemma 10. For every semi-pre-C*-algebra $\mathcal{A}$ and every $n$, one has

$$
\mathrm{C}_{\mathrm{u}}^{*}\left(\mathbb{M}_{n}(\mathcal{A})\right)=\mathbb{M}_{n}\left(\mathrm{C}_{\mathrm{u}}^{*}(\mathcal{A})\right) \text { and } \operatorname{arch}\left(\mathbb{M}_{n}(\mathcal{A})_{+}\right)=\mathbb{M}_{n}(\mathcal{A})_{+}^{\prime} .
$$

12. Completely positive maps. Completely positive maps are essential tools in study of positivity and tensor products. A linear map $\varphi: \mathcal{A} \rightarrow \mathcal{B}$ between semi-pre- $\mathrm{C}^{*}$-algebras is said to be completely positive (c.p.) if it is self-adjoint and id $\otimes \varphi: \mathbb{M}_{n}(\mathcal{A}) \rightarrow \mathbb{M}_{n}(\mathcal{B})$ is positive for every $n$. It is not too hard to see that states and positive $*$-homomorphisms are c.p.

We take back the definition of the complexification $\mathcal{A}_{\mathbb{C}}$ of a real semi-pre-C ${ }^{*}$-algebra $\mathcal{A}_{\mathbb{R}}$ and redefine

$$
\left(\mathcal{A}_{\mathbb{C}}\right)_{+}=\left\{x+\dot{\mathrm{i}} y: x \in\left(\mathcal{A}_{\mathbb{R}}\right)_{+}, y=-y^{*} \in \mathcal{A}_{\mathbb{R}} \text { such that }\left[\begin{array}{cc}
x & y \\
-y & x
\end{array}\right] \geq 0\right\} .
$$

Since this coincides with the previous definition in case of $\mathrm{C}^{*}$-algebras, they also coincide for semi-pre- $\mathrm{C}^{*}$-algebras modulo archimedean closures. The reason of this awkward replacement is to assure the following lemma holds true.

Lemma 11. Let $\varphi_{\mathbb{R}}: \mathcal{A}_{\mathbb{R}} \rightarrow \mathcal{B}_{\mathbb{R}}$ be a self-adjoint map between real semi-pre-C'-algebras. Then, its complexification $\varphi_{\mathbb{C}}: \mathcal{A}_{\mathbb{C}} \rightarrow \mathcal{B}_{\mathbb{C}}$ is c.p. if and only if $\varphi_{\mathbb{R}}$ is so.

The GNS construction for states generalizes to the Stinespring Dilation Theorem for c.p. maps. 
Theorem 12. Let $\mathcal{A}$ be a semi-pre-C*-algebra and $\varphi: \mathcal{A} \rightarrow \mathbb{B}(H)$ be a c.p. map, then there are a Hilbert space $\hat{H}$, a positive *-representation $\pi: \mathcal{A} \rightarrow \mathbb{B}(\hat{H})$, and $V \in \mathbb{B}(H, \hat{H})$ such that

$$
\varphi(a)=V^{*} \pi(a) V .
$$

for $a \in \mathcal{A}$. In particular, if $\varphi$ is unital, then $V$ is an isometry.

Proof. We introduce the semi-inner product on the algebraic tensor product $\mathcal{A} \otimes H$ by $\langle y \otimes \eta, x \otimes \xi\rangle=\left\langle\varphi\left(x^{*} y\right) \eta, \xi\right\rangle$ (and extended by linearity). That

$$
\left\langle\sum_{j} x_{j} \otimes \xi_{j}, \sum_{i} x_{i} \otimes \xi_{i}\right\rangle=\left\langle\left[\varphi\left(x_{i}^{*} x_{j}\right)\right]_{i, j} \underline{\xi}, \underline{\xi}\right\rangle \geq 0, \text { where } \underline{\xi}=\left(\xi_{1}, \ldots, \xi_{n}\right)^{T},
$$

follows from the fact $\left[x_{i}^{*} x_{j}\right]_{i, j} \in \mathbb{M}_{n}(\mathcal{A})_{+}$. Now, $\mathcal{A} \otimes H$ gives rise to a Hilbert space $\hat{H}$ and $V \in \mathbb{B}(H, \hat{H}), \xi \mapsto 1 \otimes \xi$. The $*$-representation $\pi: \mathcal{A} \rightarrow \mathbb{B}(\hat{H}), \pi(a)(x \otimes \xi)=a x \otimes \xi$, is positive and satisfies $V^{*} \pi(a) V=\varphi(a)$.

Let $\Gamma$ be a discrete group. We say an operator-valued function $f: \Gamma \rightarrow \mathbb{B}(H)$ is of positive type if $\left[f\left(x^{-1} y\right)\right]_{x, y \in E} \in \mathbb{B}\left(\ell_{2} E\right) \otimes \mathbb{B}(H)$ is positive (and self-adjoint) for every finite subset $E \subset \Gamma$. It is not too hard to see the following holds.

Corollary 13. For $f: \Gamma \rightarrow \mathbb{B}(H)$, the following are equivalent.

(1) $f$ is of positive type.

(2) $\varphi_{f}: \mathbb{k}[\Gamma] \ni g \mapsto \sum_{t} g(t) f(t) \in \mathbb{B}(H)$ is c.p.

(3) There are a unitary representation $\pi$ of $\Gamma$ on $\hat{H}$ and $V \in \mathbb{B}(H, \hat{H})$ such that $f(t)=$ $V^{*} \pi(t) V$ for $t \in \Gamma$.

We denote by $S_{n}(\mathcal{A})$ the set of unital c.p. maps from $\mathcal{A}$ into $\mathbb{M}_{n}(\mathbb{k})$.

Theorem 14. Let $\mathcal{A}_{i}$ and $\mathcal{B}_{i}$ be semi-pre-C ${ }^{*}$-algebras and $\varphi_{i}: \mathcal{A}_{i} \rightarrow \mathcal{B}_{i}$ be c.p. maps. Then, $\varphi_{1} \otimes \varphi_{2}: \mathcal{A}_{1} \otimes \mathcal{A}_{2} \rightarrow \mathcal{B}_{1} \otimes \mathcal{B}_{2}$ is c.p. with respect to each of max-max and min-min. Moreover, $\left(\mathcal{A} \otimes{ }^{\min } \mathcal{B}\right)_{+}$coincides with

$$
\left\{c \in(A \otimes B)_{\mathrm{h}}:(\varphi \otimes \psi)(c) \geq 0 \text { for all } m, n \in \mathbb{N}, \varphi \in S_{m}(\mathcal{A}), \psi \in S_{n}(\mathcal{B})\right\} .
$$

Proof. The verification of max-max case is routine (although a bit painful). The case for min-min follows from Theorem 12. We omit the details.

The u.c.p. (unital and c.p.) maps are incorporated into the free product as well.

Theorem 15 ([Bo]). Let $\mathcal{A}_{i}$ be semi-pre-C ${ }^{*}$-algebras and $\mathcal{A}=\mathcal{A}_{1} * \mathcal{A}_{2}$ be the unital free product. Let $\psi_{i} \in S\left(\mathcal{A}_{i}\right)$ be fixed and $\mathcal{A}_{i}^{0}=\operatorname{ker} \psi_{i}$ so that

$$
\mathcal{A}=\operatorname{span}\left(\mathbb{k} 1 \cup \bigcup_{n}\left\{x_{1} \cdots x_{n}: x_{j} \in \mathcal{A}_{i_{j}}^{0}, i_{1} \neq i_{2}, i_{2} \neq i_{3}, \ldots, i_{n-1} \neq i_{n}\right\}\right) \text {. }
$$


Let $\varphi_{i}: \mathcal{A}_{i} \rightarrow \mathbb{B}(H)$ be u.c.p. maps. Then, the free product map $\varphi: \mathcal{A} \rightarrow \mathbb{B}(H)$ defined by

$$
\varphi\left(x_{1} \cdots x_{n}\right)=\varphi_{i_{1}}\left(x_{1}\right) \cdots \varphi_{i_{n}}\left(x_{n}\right)
$$

is a u.c.p. map. In particular, $\varphi_{i}$ has a joint u.c.p. extension $\varphi$.

Proof. The proof of this theorem is too complicated to reproduce it here, but we prove existence of a joint u.c.p. extension, which is essentially the only part that will be used in this note. By Theorem 12, each u.c.p. map $\varphi_{i}$ extends to a positive $*$-representation $\sigma_{i}$ on $H \oplus H_{i}$. By inflating $H_{i}$ if necessary, one may assume that there is a positive $*$-representation $\sigma_{j, i}$ of $\mathcal{A}_{j}$ on $H_{i}$ for $j \neq i$. We rewrite $\sigma_{i}$ by $\sigma_{i, i}$ and consider the $*$-representations $\pi_{i}=\bigoplus_{j} \sigma_{i, j}$ of $\mathcal{A}_{i}$ on $\hat{H}=H \oplus \bigoplus_{j} H_{j}$. They extend to a positive $*$-representation $\pi$ of $\mathcal{A}$ such that $\left.P_{H} \pi(a)\right|_{H}=\varphi_{i}(a)$ for each $i$ and $a \in \mathcal{A}_{i} \subset \mathcal{A}$.

Recall that a semi-operator system is a unital *-subspace $\mathcal{S}$ of a semi-pre-C $\mathrm{C}^{*}$-algebra $\mathcal{A}$. It is equipped with the matricial positive cone $\mathbb{M}_{n}(\mathcal{S})_{+}:=\mathbb{M}_{n}(\mathcal{S})_{\mathrm{h}} \cap \mathbb{M}_{n}(\mathcal{A})_{+}$for each $n \in \mathbb{N}$. Thus the notion of complete positivity carries over to maps between semi-operator systems. We introduce a convenient tool about the one-to-one correspondence between linear maps $\varphi: \mathcal{S} \rightarrow \mathbb{M}_{n}(\mathbb{k})$ and $\tilde{\varphi}: \mathbb{M}_{n}(\mathcal{S}) \rightarrow \mathbb{k}$, given by

$$
\tilde{\varphi}\left(\left[x_{i, j}\right]\right)=\sum_{i, j} \varphi\left(x_{i, j}\right)_{i, j} \text { and } \varphi(x)=\left[\tilde{\varphi}\left(e_{i, j} \otimes x\right)\right]_{i, j} .
$$

Lemma 16. For a semi-operator system $\mathcal{S} \subset \mathcal{A}$, one has the following.

- Under the above one-to-one correspondence, $\varphi$ is c.p. if and only if $\tilde{\varphi}$ is self-adjoint and positive.

- Let $\psi: \mathcal{S} \rightarrow \mathbb{B}(H)$ be a non-zero c.p. map. Then, for the support projection $p$ of $\psi(1)$, one has $\psi(x)=p \psi(x) p$ for every $x \in \mathcal{S}$, and there is a unique u.c.p. map $\psi^{\prime}: \mathcal{S} \rightarrow$ $\mathbb{B}(p H)$ such that $\psi(x)=\psi(1)^{1 / 2} \psi^{\prime}(x) \psi(1)^{1 / 2}$. Moreover, for every $x \in \mathbb{M}_{n}(\mathbb{k}) \otimes \mathcal{S}$ one has $(\mathrm{id} \otimes \psi)(x) \geq 0$ if and only if $\left(\mathrm{id} \otimes \psi^{\prime}\right)(x) \geq 0$.

Proof. It is routine to see that $\varphi$ is self-adjoint if and only if $\tilde{\varphi}$ is. Now, for $\zeta=\sum_{i} \delta_{i} \otimes \delta_{i} \in$ $\ell_{2}^{n} \otimes \ell_{2}^{n}$, one has $\tilde{\varphi}(x)=\langle(\mathrm{id} \otimes \varphi)(x) \zeta, \zeta\rangle$. This proves $\tilde{\varphi}$ is positive if id $\otimes \varphi: \mathbb{M}_{n}(\mathcal{S}) \rightarrow$ $\mathbb{M}_{n}\left(\mathbb{M}_{n}(\mathbb{k})\right)$ is positive. The converse follows from the identity $\varphi(x)=(\mathrm{id} \otimes \tilde{\varphi})\left(\sum e_{i, j} \otimes\right.$ $\left.e_{i, j} \otimes x\right)$ and the fact that every self-adjoint positive linear functional is c.p.

For the second assertion, we first observe that for $s, t \in \mathbb{B}(H)$ one has $\left[\begin{array}{cc}s & t^{*} \\ t & 1\end{array}\right] \geq 0$ if and only if $t^{*} t \leq s$ (see Lemma 3.1 in $[\mathrm{Pa}]$ ). Let $x \in \mathcal{S} \subset \mathcal{A}$ be an element such that $x x^{*} \leq R^{2} 1$. Then,

$$
\left[\begin{array}{cc}
R & x^{*} \\
x & R
\end{array}\right]=\frac{1}{R}\left[\begin{array}{l}
R \\
x
\end{array}\right]\left[\begin{array}{ll}
R & x^{*}
\end{array}\right]+\frac{1}{R}\left[\begin{array}{l}
0 \\
1
\end{array}\right]\left(R^{2}-x x^{*}\right)\left[\begin{array}{ll}
0 & 1
\end{array}\right] \in\left(\mathbb{M}_{2}(\mathbb{k}) \otimes \mathcal{A}\right)_{+} .
$$

It follows that $\left[\begin{array}{cc}R \psi(1) & \psi(x)^{*} \\ \psi(x) & R \psi(1)\end{array}\right] \geq 0$. This implies that $\psi(x) p=\psi(x)$ for the support projection $p$ of $\psi(1)$ and

$$
\left\|(\psi(1)+\varepsilon 1)^{-1 / 2} \psi(x)(\psi(1)+\varepsilon 1)^{-1 / 2}\right\| \leq R
$$


for every $\varepsilon>0$. We define $\psi^{\prime}: \mathcal{S} \rightarrow \mathbb{B}(p H)$ to be a point-ultraweak limit point of the maps $(\psi(1)+\varepsilon 1)^{-1 / 2} \psi(\cdot)(\psi(1)+\varepsilon 1)^{-1 / 2}$ as $\varepsilon \searrow 0$. (This actually converges in norm.) This completes the proof.

Theorem 17 (Arveson's Extension Theorem). Let $\mathcal{A}$ be a semi-pre-C'-algebra and $\mathcal{S} \subset \mathcal{A}$ be a semi-operator system. Then, every c.p. map $\varphi: \mathcal{S} \rightarrow \mathbb{B}(H)$ extends to a c.p. map $\bar{\varphi}: \mathcal{A} \rightarrow \mathbb{B}(H)$.

Proof. We first deal with the case $H=\ell_{2}^{n}$. In this case, by Lemma 16, the c.p. map $\varphi: \mathcal{S} \rightarrow \mathbb{M}_{n}(\mathbb{k})$ corresponds to a positive self-adjoint linear functional $\tilde{\varphi}$ on $\mathbb{M}_{n}(\mathcal{S})$. By Corollary 4 , it extends to a positive self-adjoint linear functional on $\mathbb{M}_{n}(\mathcal{A})$. Using the correspondence again, one obtains a c.p. extension $\bar{\varphi}$ on $\mathcal{A}$.

Now, let $H$ be arbitrary. We denote by $\left\{H_{i}\right\}_{i \in I}$ the directed set of the finite-dimensional subspaces of $H$, and by $\Theta_{i}: \mathbb{B}(H) \rightarrow \mathbb{B}\left(H_{i}\right)$ the corresponding compression. From the above, each $\Theta_{i} \circ \varphi$ has a c.p. extension $\bar{\varphi}_{i}: \mathcal{A} \rightarrow \mathbb{B}\left(H_{i}\right)$. Since $\left\|\bar{\varphi}_{i}\right\| \leq\|\varphi(1)\|$ for all $i$, one may find a limit point $\bar{\varphi}$ of $\bar{\varphi}_{i}$ in the point-ultraweak topology. It is not too hard to verify that $\bar{\varphi}$ is a c.p. extension of $\varphi$.

Theorem 18 (Kadison-Choi Inequality and Choi's Multiplicative Domain). Let $\mathcal{A}$ be a semi-pre-C*-algebra and $\varphi: \mathcal{A} \rightarrow \mathbb{B}(H)$ be a u.c.p. map. Then, for every $x, y \in \mathcal{A}$, one has $\varphi\left(x^{*} x\right) \geq \varphi(x)^{*} \varphi(x)$ and

$$
\left\|\varphi\left(y^{*} x\right)-\varphi(y)^{*} \varphi(x)\right\| \leq\left\|\varphi\left(y^{*} y\right)-\varphi(y)^{*} \varphi(y)\right\|^{1 / 2}\left\|\varphi\left(x^{*} x\right)-\varphi(x)^{*} \varphi(x)\right\|^{1 / 2} .
$$

In particular,

$$
\operatorname{md}(\varphi)=\left\{x \in \mathcal{A}: \varphi\left(x^{*} x\right)=\varphi(x)^{*} \varphi(x) \text { and } \varphi\left(x x^{*}\right)=\varphi(x) \varphi(x)^{*}\right\}
$$

is a unital *-subalgebra of $\mathcal{A}$ and $\left.\varphi\right|_{\operatorname{md}(\varphi)}$ is a positive *-homomorphism.

Proof. This follows from the Stinespring Dilation Theorem. Since $\varphi$ can be expressed as $\varphi(x)=V^{*} \pi(x) V$, one has

$$
\varphi\left(x^{*} x\right)-\varphi(x)^{*} \varphi(x)=V^{*} \pi(x)^{*}\left(1-V V^{*}\right) \pi(x) V \geq 0
$$

and

$$
\varphi\left(y^{*} x\right)-\varphi(y)^{*} \varphi(x)=\left(\left(1-V V^{*}\right)^{1 / 2} \pi(y) V\right)^{*}\left(\left(1-V V^{*}\right)^{1 / 2} \pi(x) V\right) .
$$

The rest is trivial.

Theorem 19 (Pisier's Linearization Trick). Let $\mathcal{S} \subset \mathcal{A}$ be a semi-operator system, $B$ be a $\mathrm{C}^{*}$-algebra, and $\varphi: \mathcal{S} \rightarrow B$ be a u.c.p. map. Assume that

$$
\left\{x \in \mathcal{S}: 1-x^{*} x \in \operatorname{arch}\left(\mathcal{A}_{+}\right) \text {and } \varphi(x) \in B_{\mathrm{u}}\right\}
$$

generates $\mathcal{A}$ as a $*$-algebra. Then, $\varphi$ extends to a positive $*$-homomorphism from $\mathcal{A}$ into $B$. 
Proof. Let $B \subset \mathbb{B}(H)$. By Theorem $17, \varphi$ extends to a u.c.p. map $\bar{\varphi}: \mathcal{A} \rightarrow \mathbb{B}(H)$. If $x$ is in the set described in the theorem, then by Theorem 18 one has

$$
1=\bar{\varphi}(x)^{*} \bar{\varphi}(x) \leq \bar{\varphi}\left(x^{*} x\right) \leq 1,
$$

which implies $\bar{\varphi}(x)^{*} \bar{\varphi}(x)=\bar{\varphi}\left(x^{*} x\right)$. Likewise for $x^{*}$, and one has $x \in \operatorname{md}(\bar{\varphi})$. Thus, $\bar{\varphi}$ is multiplicative on $\mathcal{A}$ and it maps into $B$.

Corollary 20. Let $\mathcal{S} \subset \mathcal{A}$ be a semi-operator system which contains enough unitary elements of $\mathcal{A}$ to generate $\mathcal{A}$ as a $*$-algebra. Then, every $*$-homomorphism $\pi$ from $\mathcal{A}$ into a $\mathrm{C}^{*}$-algebra $B$ is positive provided that $\left.\pi\right|_{\mathcal{S}}$ is c.p. Moreover,

$$
\operatorname{arch}\left(\mathcal{A}_{+}\right)=\operatorname{arch}\left(\left\{\sum_{i, j} x_{i}^{*} a_{i, j} x_{j}: n \in \mathbb{N},\left[a_{i, j}\right]_{i, j} \in \mathbb{M}_{n}(\mathcal{S})_{+}, x_{1}, \ldots, x_{n} \in \mathcal{A}\right\}\right) .
$$

Proof. The first assertion is immediate. For the second, observe that the right hand side defines a $*$-positive cone on the $*$-algebra $\mathcal{A}$, which gives rise to the same universal $\mathrm{C}^{*}$-algebra as that of the original $\mathcal{A}$.

13. Kirchberg's Theorem on $\mathrm{C}^{*} \mathbb{F}_{2} \otimes \mathbb{B}\left(\ell_{2}\right)$. We will prove the following celebrated theorem of Kirchberg. The proof takes a similar line as [Pi1, FP].

Theorem 21 ([Ki1]). Let $A_{i}$ be $\mathrm{C}^{*}$-algebras and $A=A_{1} * A_{2}$ be their unital full free product $\mathrm{C}^{*}$-algebra. If $A_{i} \otimes_{\max } \mathbb{B}\left(\ell_{2}\right)=A_{i} \otimes_{\min } \mathbb{B}\left(\ell_{2}\right)$ for each $i$, then $A \otimes_{\max } \mathbb{B}\left(\ell_{2}\right)=A \otimes_{\min } \mathbb{B}\left(\ell_{2}\right)$. In particular, one has

$$
\mathrm{C}^{*} \mathbb{F}_{d} \otimes_{\max } \mathbb{B}(H)=\mathrm{C}^{*} \mathbb{F}_{d} \otimes_{\min } \mathbb{B}(H)
$$

for every $d$ and every Hilbert space $H$.

The proof requires a description of the operator system structure of $A_{1}+A_{2} \subset A$.

Lemma 22 ([Ka]). Let $\mathcal{A}=\mathcal{A}_{1} * \mathcal{A}_{2}$ be a unital full free product and $\mathcal{S}=\mathcal{A}_{1}+\mathcal{A}_{2} \subset \mathcal{A}$ be the semi-operator system. Then, $a \in \mathcal{S} \otimes \mathbb{B}\left(\ell_{2}\right)$ belongs to $\left(\mathcal{A} \otimes{ }^{\min } \mathbb{B}\left(\ell_{2}\right)\right)_{+}$if and only if there are $a_{i} \in\left(\mathcal{A}_{i} \otimes \min \mathbb{B}\left(\ell_{2}\right)\right)_{+}$such that $a=a_{1}+a_{2}$.

Proof. The "if" direction is trivial. For the other direction, we first deal with the case $a \in\left(\mathcal{S} \otimes{ }^{\min } \mathbb{B}\left(\ell_{2}^{n}\right)\right)_{+}$. Let us consider the cone

$$
C=\left\{a_{1}+a_{2}: a_{i} \in\left(\mathcal{A}_{i} \otimes^{\min } \mathbb{B}\left(\ell_{2}^{n}\right)\right)_{+}\right\} .
$$

Since $C$ is algebraically solid, if $a \notin \operatorname{arch}(C)$, then there is a self-adjoint linear functional $\varphi: \mathcal{S} \otimes \mathbb{B}\left(\ell_{2}^{n}\right) \rightarrow \mathbb{k}$ such that

$$
\varphi(a)<0 \leq \inf _{c \in C} \varphi(c) .
$$

Then, $\varphi_{i}=\left.\varphi\right|_{\mathcal{A}_{i} \otimes \min \mathbb{B}\left(\ell_{2}^{n}\right)}$ are self-adjoint positive linear functionals, and by Lemma 16 they correspond to c.p. maps $\psi_{i}: \mathcal{A}_{i} \rightarrow \mathbb{B}\left(\ell_{2}^{n}\right)$. Let $p$ be the support projection of $h=$ $\psi_{1}(1)=\psi_{2}(1)$. Then the maps $\psi_{i}^{\prime}(\cdot)=\left.h^{-1 / 2} \psi_{i}(\cdot) h^{-1 / 2}\right|_{p \ell_{2}^{n}}$ are u.c.p. and extend to a u.c.p. map $\psi^{\prime}$ on $\mathcal{A}$ by Theorem 15. It follows that $\left(h^{1 / 2} \otimes 1\right)\left(\psi^{\prime} \otimes \operatorname{id}(a)\right)\left(h^{1 / 2} \otimes 1\right) \geq 0$. 
This implies $\varphi(a) \geq 0$ in contradiction with the hypothesis. Thus, we have shown that $\left(\mathcal{S} \otimes{ }^{\min } \mathbb{B}\left(\ell_{2}^{n}\right)\right)_{+} \subset \operatorname{arch}(C)$.

Now, let $a \in\left(\mathcal{S} \otimes{ }^{\text {min }} \mathbb{B}\left(\ell_{2}\right)\right)_{+}$. By the previous result, for every $n$, there are $a_{i}(n) \in$ $\left(\mathcal{A}_{i} \otimes{ }^{\min } \mathbb{B}\left(\ell_{2}^{n}\right)\right)_{+}$such that $\left(\mathrm{id} \otimes \Theta_{n}\right)(a+1 / n)=a_{1}(n)+a_{2}(n)$, where $\Theta_{n}: \mathbb{B}\left(\ell_{2}\right) \rightarrow \mathbb{B}\left(\ell_{2}^{n}\right)$ is the compression. For $R>0$ such that $R 1 \geq a$, one has $0 \leq a_{i}(n) \leq(R+1) 1$ for all $n$. Take unital finite-dimensional subspaces $E_{i} \subset \mathcal{A}_{i}$ such that $a \in\left(E_{1}+E_{2}\right) \otimes \mathbb{B}\left(\ell_{2}\right)$. Since $\mathcal{A}_{1} \cap \mathcal{A}_{2}=\mathbb{k} 1$, one has $a_{i}(n) \in E_{i} \otimes \mathbb{B}\left(\ell_{2}^{n}\right)$. Therefore, one may find ultraweak limit points $a_{i}$ of $\left(a_{i}(n)\right)_{n=1}^{\infty}$ (jointly for $\left.i=1,2\right)$ in $E_{i} \otimes \mathbb{B}\left(\ell_{2}\right)$. It follows that $a_{i} \geq 0$ and $a=a_{1}+a_{2}$.

Proof of Theorem 21. By Corollary 20, it suffices to show that the formal identity map from $A \otimes{ }^{\min } \mathbb{B}\left(\ell_{2}\right)$ into $A \otimes_{\max } \mathbb{B}\left(\ell_{2}\right)$ is c.p. on $\mathcal{S} \otimes \mathbb{B}\left(\ell_{2}\right)$, where $\mathcal{S}=A_{1}+A_{2} \subset A$. Since $\mathbb{M}_{n}(\mathbb{k}) \otimes \mathbb{B}\left(\ell_{2}\right) \cong \mathbb{B}\left(\ell_{2}\right)$, we only have to show that it is positive. But if $a \in \mathcal{S} \otimes \mathbb{B}\left(\ell_{2}\right)$ is positive, then $a=a_{1}+a_{2}$ for some $a_{i} \in\left(A_{i} \otimes^{\min } \mathbb{B}\left(\ell_{2}\right)\right)_{+}$by the above lemma. By assumption, one has $a_{i} \in \operatorname{arch}\left(A_{i} \otimes{ }^{\max } \mathbb{B}\left(\ell_{2}\right)\right)_{+}$. Now, it is easy to see that $a=a_{1}+a_{2} \in$ $\operatorname{arch}\left(A \otimes{ }^{\max } \mathbb{B}\left(\ell_{2}\right)\right)$. This completes the proof.

14. The positive cone of the free group algebras. Schmüdgen has proved in 1980s (unpublished, see $[\mathrm{NT}]$ ) that $\mathbb{k}\left[\mathbb{F}_{d}\right]_{+}$is archimedean closed. It was generalized by McCullough $([\mathrm{Mc}])$ and Bakonyi-Timotin $([\mathrm{BT}])$ to the operator-valued case. Here we give a somewhat simpler proof of this result. Our proof employs a well-known matrix completion trick, in conjunction with the geometry of a tree.

For notational simplicity, we fix a Hilbert space $H$ throughout this section and denote $\mathbb{B}=\mathbb{B}(H)$. In particular, $\mathbb{B}=\mathbb{k}$ when $\operatorname{dim} H=1$. We denote by $\mathbb{B}[\Gamma]$ the group algebra of $\Gamma$ with coefficients in $\mathbb{B}$. So, we will view an element $f \in \mathbb{B}[\Gamma]$ as a function $f: \Gamma \rightarrow \mathbb{B}$. One has

$$
\mathbb{B}[\Gamma] \cong \mathbb{B}(H) \otimes^{\max } \mathbb{k}[\Gamma] \text { and } \mathbb{B}[\Gamma]_{+}=\left\{\sum_{i=1}^{n} \xi_{i}^{*} * \xi_{i}: n \in \mathbb{N}, \xi_{i} \in \mathbb{B}[\Gamma]\right\} .
$$

We first characterize those elements which belong to $\mathbb{B}[\Gamma]_{+}$. We denote by $\rho$ the right regular representation of $\Gamma$ on $\ell_{2} \Gamma: \rho(a) \delta_{t}=\delta_{t a^{-1}}$ for $a, t \in \Gamma$; and for a finite subset $E \subset \Gamma$, by $\rho_{E}(a)$ the compression of $\rho(a)$ to $\mathbb{B}\left(\ell_{2} E\right)$. We identify $\mathbb{B}\left(\ell_{2} E\right)$ with $\mathbb{M}_{E}(\mathbb{k})$ and let $\mathcal{T}_{E} \subset \mathbb{M}_{E}(\mathbb{k})$ be the operator system consisting of the "Toeplitz operators,"

$$
\begin{aligned}
\mathcal{T}_{E} & =\left\{\rho_{E}(g): g \in \mathbb{k}[\Gamma]\right\} \\
& =\left\{X \in \mathbb{M}_{E}(\mathbb{k}): \exists g \in \mathbb{k}[\Gamma] \text { such that } X=\left[g\left(s^{-1} t\right)\right]_{s, t \in E}\right\} .
\end{aligned}
$$

Lemma 23. Let $f \in \mathbb{B}[\Gamma]_{\mathrm{h}}$ and $E \subset \Gamma$ be a finite subset such that $\operatorname{supp} f \subset E^{-1} E$. Then, $f \in\left\{\sum_{i=1}^{n} \xi_{i}^{*} * \xi_{i}: n \in \mathbb{N}, \xi_{i} \in \mathbb{B}[E]\right\}$ if and only if the linear map $\varphi_{f}: \mathcal{T}_{E} \rightarrow \mathbb{B}$, given by $\varphi_{f}\left(\rho_{E}(a)\right)=f(a)$, is c.p. One may take $n \leq|E|$ in the above factorization of $f$, and if $\operatorname{dim} \mathbb{B}=\infty$ in addition, then one may take $n=1$. 
Proof. If $f=\sum_{i} \xi_{i}^{*} * \xi_{i}$, then for $X=\left[X_{s, t}\right]_{s, t}=\rho_{E}(g) \in \mathcal{T}_{E}$, one has

$$
\varphi_{f}(X)=\sum_{a} g(a) f(a)=\sum_{i} \sum_{a, t} g(a) \xi_{i}^{*}\left(a t^{-1}\right) \xi_{i}(t)=\sum_{i} \sum_{s, t} X_{s, t} \xi_{i}(s)^{*} \xi_{i}(t) .
$$

From the latter expression, it is not too hard to see $\varphi_{f}$ is c.p.

Conversely, if $\varphi_{f}$ is c.p., then it extends to a c.p. map, still denoted by $\varphi_{f}$, on $\mathbb{M}_{E}\left(\mathbb{k}_{k}\right)$ by Theorem 17. It follows that $b=\left[\varphi_{f}\left(e_{s, t}\right)\right]_{s, t \in E}$ is positive in $\mathbb{M}_{E}(\mathbb{B})$. Develop $b^{1 / 2}$ as $\left[\xi_{i}(t)\right]_{i, t \in E}$. Then, one has

$$
\left(\sum_{i} \xi_{i}^{*} * \xi_{i}\right)(a)=\sum_{i} \sum_{t} \xi_{i}\left(t a^{-1}\right)^{*} \xi_{i}(t)=\sum_{t} b_{t a^{-1}, t}=\varphi_{f}\left(\rho_{E}(a)\right)=f(a) .
$$

We note that if $\operatorname{dim} \mathbb{B}=\infty$, then there are isometries $S_{i} \in \mathbb{B}$ with mutually orthogonal ranges, and $\xi=\sum_{i} S_{i} \xi_{i} \in \mathbb{B}[E]$ satisfies $f=\xi^{*} * \xi$.

We need the following well-known matrix completion trick.

Lemma 24. Let $H_{0} \oplus H_{1} \oplus H_{2}$ be a direct sum of Hilbert spaces. Suppose

$$
\left[\begin{array}{ccc}
A & X & ? \\
X^{*} & B & Y \\
? & Y^{*} & C
\end{array}\right] \in \mathbb{B}\left(H_{0} \oplus H_{1} \oplus H_{2}\right)
$$

is a partially defined operator matrix such that its compressions on $H_{0} \oplus H_{1}$ and $H_{1} \oplus H_{2}$ are both positive. Then one can complete the unspecified block ? so that the resultant operator matrix is positive on $H_{0} \oplus H_{1} \oplus H_{2}$.

Proof. We note that $\left[\begin{array}{cc}A & X \\ X^{*} & B\end{array}\right] \geq 0$ if and only if $X_{1}:=A^{-1 / 2} X B^{-1 / 2}$ is well-defined and contractive (see Lemma 3.1 in $[\mathrm{Pa}]$ ). Likewise for $Y_{1}:=B^{-1 / 2} Y C^{-1 / 2}$. Now, since

$$
\left[\begin{array}{ccc}
1 & X_{1} & X_{1} Y_{1} \\
X_{1}^{*} & 1 & Y_{1} \\
Y_{1}^{*} X_{1}^{*} & Y_{1}^{*} & 1
\end{array}\right]=\left[\begin{array}{ccc}
1 & 0 & 0 \\
X_{1}^{*} & \left(1-X_{1}^{*} X_{1}\right)^{1 / 2} & 0 \\
Y_{1}^{*} X_{1}^{*} & Y_{1}^{*}\left(1-X_{1}^{*} X_{1}\right)^{1 / 2} & \left(1-Y_{1}^{*} Y_{1}\right)^{1 / 2}
\end{array}\right]\left[\begin{array}{ccc}
1 & X_{1} & X_{1} Y_{1} \\
0 & \left(1-X_{1}^{*} X_{1}\right)^{1 / 2} & \left(1-X_{1}^{*} X_{1}\right)^{1 / 2} Y_{1} \\
0 & 0 & \left(1-Y_{1}^{*} Y_{1}\right)^{1 / 2}
\end{array}\right],
$$

the operator matrix

$$
\left[\begin{array}{ccc}
A & X & Z \\
X^{*} & B & Y \\
Z^{*} & Y^{*} & C
\end{array}\right]=\left[\begin{array}{ccc}
A^{1 / 2} & 0 & 0 \\
0 & B^{1 / 2} & 0 \\
0 & 0 & C^{1 / 2}
\end{array}\right]\left[\begin{array}{ccc}
1 & X_{1} & X_{1} Y_{1} \\
X_{1}^{*} & 1 & Y_{1} \\
Y_{1}^{*} X_{1}^{*} & Y_{1}^{*} & 1
\end{array}\right]\left[\begin{array}{ccc}
A^{1 / 2} & 0 & 0 \\
0 & B^{1 / 2} & 0 \\
0 & 0 & C^{1 / 2}
\end{array}\right]
$$

is positive for $Z=X B^{-1} Y$.

Let $s_{1}, \ldots, s_{d}$ be the canonical generators of the free group $\mathbb{F}_{d}$. A pair $\{s, t\}$ in $\mathbb{F}_{d}$ is adjacent in the Cayley graph of $\mathbb{F}_{d}$ if $s t^{-1} \in\left\{s_{1}^{ \pm 1}, \ldots, s_{d}^{ \pm 1}\right\}$. The Cayley graph of $\mathbb{F}_{d}$ is a simplicial tree. We say a subset $E \subset \mathbb{F}_{d}$ is grounded if it contains the unit 1 and is connected in the Cayley graph. Thus, $E$ is grounded if and only if $t \in E$ and $t=t^{\prime} t^{\prime \prime}$ without cancelation imply $t^{\prime \prime} \in E$.

Lemma 25 (Proposition 4.4 in $[\mathrm{BT}]$ ). Let $g \in \mathbb{B}\left[\mathbb{F}_{d}\right]_{\mathrm{h}}$ and $E \subset \mathbb{F}_{d}$ be a finite grounded subset. If $\left(\rho_{E} \otimes \operatorname{id}_{\mathbb{B}}\right)(g) \geq 0$ in $\mathbb{M}_{E}(\mathbb{B})$, then there is a positive type function $g^{\prime}: \mathbb{F}_{d} \rightarrow \mathbb{B}$ such that $g^{\prime}=g$ on $E^{-1} E$. 
Proof. By inductive construction, it suffices to show that for every grounded subset $E^{\prime} \supset$ $E$ such that $\left|E^{\prime} \backslash E\right|=1$, one can find $g^{\prime} \in \mathbb{B}\left[\mathbb{F}_{d}\right]_{\mathrm{h}}$ such that $g^{\prime}=g$ on $E^{-1} E$ and $\left(\rho_{E^{\prime}} \otimes \operatorname{id}_{\mathbb{B}}\right)\left(g^{\prime}\right) \geq 0$. Thus, let $E^{\prime}=E \cup\left\{t_{0}\right\}$ with $t_{0}=s_{i} t_{0}^{\prime \prime}$ for $t_{0}^{\prime \prime} \in E$ and one of the generators $s_{i}$. (The case for $t_{0}=s_{i}^{-1} t_{0}^{\prime \prime}$ is similar.) We claim that

$$
\left\{s \in E: s^{-1} t_{0} \in E^{-1} E\right\}=\left\{s \in E: s_{i}^{-1} s \in E\right\} .
$$

Indeed, if $s=s_{i} s^{\prime \prime}$ for some $s^{\prime \prime} \in E$, then $s^{-1} t_{0}=\left(s^{\prime \prime}\right)^{-1} t_{0}^{\prime \prime} \in E^{-1} E$. To prove the converse inclusion, suppose $s^{-1} t_{0} \in E^{-1} E$. By groundedness, there is a non-trivial decomposition $t_{0}=p q$ without cancelation such that $p^{-1} s \in E$. Note that the first letter of $p$ is $s_{i}$. If the reduced form of $s$ starts by $s_{i}$, then $s_{i}^{-1} s \in E$ by groundedness. Otherwise, there is no cancelation between $p^{-1}$ and $s$, and hence $p^{-1} s \in E$ implies $s_{i}^{-1} s \in E$ by groundedness. The claim is proved. We denote the above subset by $E_{1}$ and set $E_{0}=E \backslash E_{1}$.

Now we extend $\rho_{E}(g)$ to a partially defined matrix $X=\left[X_{s, t}\right]_{s, t \in E^{\prime}}$ such that $X_{s, t}=$ $g\left(s^{-1} t\right)$ for $(s, t) \in E^{\prime} \times E^{\prime}$ with $s^{-1} t \in E^{-1} E$. The unspecified entries of $X$ are those for $\left(s, t_{0}\right)$ and $\left(t_{0}, s\right)$ with $s \in E_{0}$. We apply Lemma 24 to $H_{0}=\ell_{2} E_{0}, H_{1}=\ell_{2} E_{1}$, and $H_{2}=\ell_{2}\left\{t_{0}\right\}$. We note that the compression of $X$ on $H_{1} \oplus H_{2}$ is positive, thanks to the right equivariant map $E_{1} \cup\left\{t_{0}\right\} \ni s \mapsto s_{i}^{-1} s \in E$. Thus, one obtains a fully defined positive matrix $X$. We observe that $s^{-1} t_{0} \neq t_{0}^{-1} s^{\prime}$ for any $s, s^{\prime} \in E_{0}$. Indeed, the shortest right segments that does not belong to $E$ is $s_{i} t_{0}^{\prime \prime}$ in the left hand side and $s_{i}^{-1} s^{\prime}$ in the right hand side. It follows that the entry $X_{s, t}$ of the positive matrix $X \in \mathbb{M}_{E^{\prime}}(\mathbb{B})$ depends only on $s^{-1} t$ and $X$ is of the form $\left(\rho_{E^{\prime}} \otimes \mathrm{id}_{\mathbb{B}}\right)\left(g^{\prime}\right)$.

The following is slightly more precise than Theorem 7.1 in [BT] (and Theorem 0.1 in $[\mathrm{Mc}])$. It implies $\mathbb{B}(H) \otimes^{\max } \mathbb{k}\left[\mathbb{F}_{d}\right]=\mathbb{B}(H) \otimes^{\min } \mathbb{k}\left[\mathbb{F}_{d}\right]$, and hence Theorem 21 .

Theorem $26([\mathrm{BT}])$. Let $\mathbb{B}=\mathbb{B}(H), f \in \mathbb{B}_{\mathbb{F}}\left[\mathbb{F}_{\mathrm{h}}\right.$, and $E \subset \mathbb{F}_{d}$ be a grounded subset such that $\operatorname{supp} f \subset E^{-1} E$. Assume that $\left(\operatorname{id}_{\mathbb{B}} \otimes \pi\right)(f) \geq 0$ for every finite-dimensional unitary representation $\pi$ of $\mathbb{F}_{d}$ (of dimension at most $2|E| \operatorname{dim} H$ ). Then, there are $n \leq|E|$ and $\xi_{1}, \ldots, \xi_{n} \in \mathbb{B}\left[\mathbb{F}_{d}\right]$ such that $\operatorname{supp} \xi_{i} \subset E$ and $f=\sum_{i=1}^{n} \xi_{i}^{*} * \xi_{i}$. If $\operatorname{dim} \mathbb{B}=\infty$ in addition, then one may take $n=1$.

Proof. By Lemma 23 (we may assume $E$ is finite), it suffices to show $\varphi_{f}: \mathcal{T}_{E} \rightarrow \mathbb{B}(H)$ is c.p. Let $g: \mathbb{F}_{d} \rightarrow \mathbb{M}_{k}\left(\mathbb{k}_{k}\right)$ be such that $\left(\rho_{E} \otimes \mathrm{id}\right)(g) \geq 0$. By Lemma 25 , we may assume that $g$ is of positive type. Hence, by Corollary 13, there are a unitary representation $\sigma$ on $H_{\sigma}$ and $V \in \mathbb{B}\left(\ell_{2}^{k}, H_{\sigma}\right)$ such that $g(a)=V^{*} \sigma(a) V$. One has to show that

$$
\left(\varphi_{f} \otimes \mathrm{id}\right)\left(\left(\rho_{E} \otimes \mathrm{id}\right)(g)\right)=\sum_{a \in E^{-1} E} f(a) \otimes g(a)=(1 \otimes V)^{*}\left(\left(\mathrm{id}_{\mathbb{B}} \otimes \sigma\right)(f)\right)(1 \otimes V)
$$

is positive. We will follow Choi's proof of RFD for $\mathrm{C}^{*} \mathbb{F}_{d}$. Suppose the contrary that $\left(\operatorname{id}_{\mathbb{B}} \otimes \sigma\right)(f)$ is not positive. Then, there is a vector $\zeta$ in the algebraic tensor product $H \otimes H_{\sigma}$ such that $\left\langle\left(\operatorname{id}_{\mathbb{B}} \otimes \sigma\right)(f) \zeta, \zeta\right\rangle<0$. We may find a finite-dimensional subspace $H_{0} \subset H_{\sigma}$ of dimension at most $\operatorname{dim} H$ such that $\zeta \in H \otimes H_{0}$. Let $H_{1}=\operatorname{span} \sigma(E) H_{0}$ and 
write $X_{i}=\left.P_{H_{1}} \sigma\left(s_{i}\right)\right|_{H_{1}} \in \mathbb{B}\left(H_{1}\right)$ for the generators $s_{1}, \ldots, s_{d} \in \mathbb{F}_{d}$. We define the unitary representation $\pi$ of $\mathbb{F}_{d}$ by assigning to each $s_{i}$ a unitary element

$$
\pi\left(s_{i}\right)=\left[\begin{array}{cc}
X_{i} & \left(1-X_{i} X_{i}^{*}\right)^{1 / 2} \\
\left(1-X_{i}^{*} X_{i}\right)^{1 / 2} & -X_{i}^{*}
\end{array}\right] \in \mathbb{M}_{2}\left(\mathbb{B}\left(H_{1}\right)\right)_{\mathrm{u}} .
$$

It follows that $(1 \otimes \pi)(t)(\zeta \oplus 0)=(1 \otimes \sigma)(t) \zeta \oplus 0$ for every $t \in E$, and $\left\langle\left(\operatorname{id}_{\mathbb{B}} \otimes \pi\right)(f)(\zeta \oplus\right.$ $0), \zeta \oplus 0\rangle=\left\langle\left(\operatorname{id}_{\mathbb{B}} \otimes \sigma\right)(f) \zeta, \zeta\right\rangle<0$, in contradiction with the assumption.

Rudin $([\mathrm{Ru}])$ has proved existence of $f \in \operatorname{arch}\left(\mathbb{k}\left[\mathbb{Z}^{2}\right]_{+}\right)$such that $\operatorname{supp} f \subset E^{-1} E$ with $E=[0, N]^{2}$ but $f$ cannot be expressed as $\sum_{i} \xi_{i}^{*} * \xi_{i}$ for any $\xi_{i} \in \mathbb{k}[E]$. This implies that the matrix completion problem of the following type has a negative answer in general: Given Toeplitz matrices $A_{k}=A_{-k}^{*} \in \mathbb{M}_{n}(\mathbb{k}),|k| \leq m-1$, whose Toeplitz operator matrix $\left[A_{i-j}\right]_{i, j=1}^{m} \in \mathbb{M}_{m}\left(\mathbb{M}_{n}(\mathbb{k})\right)$ is positive, can one find a Toeplitz matrix $A_{m}=A_{-m}^{*} \in \mathbb{M}_{n}(\mathbb{k})$ so that $\left[A_{i-j}\right]_{i, j=1}^{m+1} \in \mathbb{M}_{m+1}\left(\mathbb{M}_{n}(\mathbb{k})\right)$ is still positive? We note that nonetheless $\mathbb{k}_{k}\left[\mathbb{Z}^{2}\right]_{+}$is archimedean closed $([\mathrm{Sd}])$.

15. Kirchberg's Conjecture. In his seminal paper [Ki1], Kirchberg has shown that the Connes Embedding Conjecture (see Section 10) is equivalent to several other important conjectures in operator algebra theory, one of which is Kirchberg's Conjecture (KC) that

$$
\mathrm{C}^{*} \mathbb{F}_{d} \otimes_{\max } \mathrm{C}^{*} \mathbb{F}_{d}=\mathrm{C}^{*} \mathbb{F}_{d} \otimes_{\min } \mathrm{C}^{*} \mathbb{F}_{d}
$$

holds for some/all $d \geq 2$. In this section, we assume the scalar field is complex, $\mathbb{k}=\mathbb{C}$, and give the proof of the equivalence. The proofs of this section are analytically involved, not so self-contained, and probably off the scope of this note, but we include them because some results seem to be new or at least not well documented in the literature. Consult [BO] for technical terms in the proofs which are not explained in this note.

Recall that the opposite algebra of an algebra $\mathcal{A}$ is the algebra

$$
\mathcal{A}^{\mathrm{op}}=\left\{a^{\mathrm{op}}: a \in \mathcal{A}\right\}
$$

which has the same linear and $*$-positive structures as $\mathcal{A}$, but has opposite multiplication $a^{\mathrm{op}} b^{\mathrm{op}}=(b a)^{\mathrm{op}}$. It is $*$-isomorphic to the complex conjugate $\overline{\mathcal{A}}$ of $\mathcal{A}$ by $a^{\mathrm{op}} \leftrightarrow \bar{a}^{*}$. We note that $\mathbb{C}[\Gamma]^{\text {op }} \cong \mathbb{C}[\Gamma]$ via $f \leftrightarrow \check{f}$, where $\check{f}(s)=f\left(s^{-1}\right)$.

Theorem 27 ([Ki1]). Let $A$ be a $\mathrm{C}^{*}$-algebra with a tracial state $\tau$, and $u_{1}, u_{2}, \ldots$ be a dense sequence in the unitary group $A_{\mathrm{u}}$ of $A$. Let $(M, \tau)$ be a finite von Neumann algebra and suppose that there is a sequence $v_{1}, v_{2}, \ldots$ of unitary elements in $M$ such that $\tau\left(u_{i}^{*} u_{j}\right)=$ $\tau\left(v_{i}^{*} v_{j}\right)$ for all $i, j$. Then, there is a projection $p \in M$ such that $\pi_{\tau}(A)^{\prime \prime}$ is embeddable into $p M p \oplus\left(p^{\perp} M p^{\perp}\right)^{\mathrm{op}}$. In particular, if $M$ moreover satisfies $p^{\perp} M p^{\perp} \cong\left(p^{\perp} M p^{\perp}\right)^{\text {op }}$ (e.g., $\left.M=R^{\omega}\right)$, then $\pi_{\tau}(A)^{\prime \prime} \hookrightarrow M$.

Proof. We reproduce Kirchberg's proof ([Ki1]) here. The map $u_{i} \mapsto v_{i}$ extends to a linear isometry $\varphi$ from $L^{2}(A, \tau)$ into $L^{2}(M, \tau)$. Since the set of unitary elements is closed in $L^{2}(M, \tau)$, the map $\varphi$ sends unitary elements to unitary elements by density and continuity. 
By the Russo-Dye theorem, $\varphi$ is a contraction from $A$ into $M$. Thus the unital map $\theta$ defined by $\theta(a)=\varphi(1)^{*} \varphi(a)$ is a positive contraction from $A$ into $M$, which sends unitary elements to unitary elements and preserves the tracial state. By a multiplicative domain argument (Corollary 2.6 in [Oz1]), the map $\theta$ is a Jordan morphism. Thus, there is a central projection $p$ in $\theta(A)^{\prime \prime}$ such that $p \theta$ (resp. $p^{\perp} \theta$ ) is a $*$-homomorphism (resp. an opposite $*$-homomorphism).

Let $\tau$ be a tracial state on a semi-pre- $\mathrm{C}^{*}$-algebra $\mathcal{A}$. Then, besides the GNS representation $\pi_{\tau}$ of $\mathcal{A}$ on $L^{2}(\mathcal{A}, \tau)$, there is a representation $\pi_{\tau}^{\text {op }}$ of $\mathcal{A}^{\text {op }}$ on $L^{2}(\mathcal{A}, \tau)$, given by $\pi_{\tau}^{\mathrm{op}}\left(b^{\mathrm{op}}\right) \hat{x}=\widehat{x b}$. Since $\tau$ is tracial, one has

$$
\left\langle\pi_{\tau}^{\mathrm{op}}\left(b^{\mathrm{op}}\right) \hat{x}, \hat{x}\right\rangle=\tau\left(x^{*} x b\right)=\left\langle\pi_{\tau}(b) \hat{x}^{*}, \hat{x}^{*}\right\rangle \geq 0
$$

if $b^{\text {op }} \geq 0$, which means that $\pi_{\tau}^{\text {op }}$ is a positive $*$-representation on $L^{2}(\mathcal{A}, \tau)$.

Since $\pi_{\tau}(\mathcal{A})$ and $\pi_{\tau}^{\mathrm{op}}\left(\mathcal{A}^{\mathrm{op}}\right)$ commute, one obtains a positive $*$-representation

$$
\pi_{\tau} \times \pi_{\tau}^{\mathrm{op}}: \mathcal{A} \otimes \mathcal{A}^{\mathrm{op}} \rightarrow \mathbb{B}\left(L^{2}(\mathcal{A}, \tau)\right),\left(\pi_{\tau} \times \pi_{\tau}^{\mathrm{op}}\right)\left(a \otimes b^{\mathrm{op}}\right) \hat{x}=\widehat{a x b} .
$$

In particular,

$$
\sum a_{i} \otimes b_{i}^{\mathrm{op}} \mapsto\left\langle\left(\pi_{\tau} \times \pi_{\tau}^{\mathrm{op}}\right)\left(\sum a_{i} \otimes b_{i}^{\mathrm{op}}\right) \hat{1}, \hat{1}\right\rangle=\tau\left(\sum a_{i} b_{i}\right)
$$

is a state on $\mathcal{A} \otimes{ }^{\max } \mathcal{A}^{\text {op }}$.

To prove the equivalence between CEC and KC, Kirchberg ([Ki1]) has shown that all von Neumann algebras are QWEP if it is the case for finite von Neumann algebras. We take a different route and prove the following alternative.

Theorem 28. There is a tracial state $\tau$ on $\mathrm{C}^{*} \mathbb{F}_{d}$ such that $\pi_{\tau} \times \pi_{\tau}^{\mathrm{op}}$ is faithful on the $\mathrm{C}^{*}$-algebra $\mathrm{C}^{*} \mathbb{F}_{d} \otimes_{\max } \mathrm{C}^{*} \mathbb{F}_{d}$. This means that if $\tau\left(\sum_{i} a_{i} x b_{i} x^{*}\right) \geq 0$ for every $x \in \mathbb{C}\left[\mathbb{F}_{d}\right]$ and $\tau \in T\left(\mathbb{C}\left[\mathbb{F}_{d}\right]\right)$, then $\sum_{i} a_{i} \otimes \check{b}_{i} \in \operatorname{arch}\left(\mathbb{C}\left[\mathbb{F}_{d} \times \mathbb{F}_{d}\right]_{+}\right)$.

Proof. First, take a faithful *-representation $\pi \times \pi^{\prime}$ of $\mathrm{C}^{*} \mathbb{F}_{d} \otimes_{\max }\left(\mathrm{C}^{*} \mathbb{F}_{d}\right)^{\text {op }}$ on $H$. By inflating it if necessary, one may find a von Neumann algebra $M$ in its standard form (see Section IX.1 in $[\mathrm{Ta}])$ such that $\pi\left(\mathrm{C}^{*} \mathbb{F}_{d}\right) \subset M$ and $\pi^{\prime}\left(\left(\mathrm{C}^{*} \mathbb{F}_{d}\right)^{\mathrm{op}}\right) \subset M^{\prime} \cong M^{\mathrm{op}}$. Further, by replacing $\pi$ with $\pi \oplus\left(\pi^{\prime}\right)^{\text {op }}: \mathrm{C}^{*} \mathbb{F}_{d} \rightarrow M \oplus M \subset \mathbb{M}_{2}(M)$, and $M$ with $\mathbb{M}_{2}(M)$, one may assume $\pi^{\prime}=\pi^{\mathrm{op}}$. There is an $\mathbb{R}$-action on $M$ such that the crossed product von Neumann algebra $M \rtimes \mathbb{R}$ is semi-finite (Theorem XII.1.1 in [Ta]). We think $M \rtimes \mathbb{R}$ acts on $H \otimes L^{2}(\mathbb{R})$ by $a \otimes 1$ for $a \in M$ and $u_{t} \otimes \lambda_{t}$ for $t \in \mathbb{R}$, where $u_{t}$ is the implementing unitary group. Hence $\mathrm{C}^{*} \mathbb{F}_{d} \hookrightarrow M \rtimes \mathbb{R}$ is given by $\tilde{\pi}(a)=\pi(a) \otimes 1$. Then under the identification of $H \otimes L^{2}(\mathbb{R})$ with $L^{2}(\mathbb{R}, H)$, the embedding $\tilde{\pi}^{\text {op }}:\left(\mathrm{C}^{*} \mathbb{F}_{d}\right)^{\text {op }} \hookrightarrow(M \rtimes \mathbb{R})^{\prime}$ is given by $\left(\tilde{\pi}^{\mathrm{op}}\left(b^{\mathrm{op}}\right) \xi\right)(t)=u_{t} \pi^{\mathrm{op}}\left(b^{\mathrm{op}}\right) u_{t}^{*} \xi(t)$. Since $\pi \times \pi^{\mathrm{op}}$ is weakly contained in $\tilde{\pi} \times \tilde{\pi}^{\mathrm{op}}$, one may assume from the beginning that $M$ is a semi-finite von Neumann algebra with a faithful normal tracial weight $\mathrm{Tr}$, and the $*$-representation $\pi \times \pi^{\mathrm{op}}$ of $\mathrm{C}^{*} \mathbb{F}_{d} \otimes_{\max }\left(\mathrm{C}^{*} \mathbb{F}_{d}\right)^{\mathrm{op}}$ on $L^{2}(M, \operatorname{Tr})$ is faithful. Since vectors with finite supports are dense in $L^{2}(M, \operatorname{Tr})$, one 
can use Choi's trick (see the proof of Theorem 26) and find a countable family of $*$-representations $\sigma_{k}$ from $\mathrm{C}^{*} \mathbb{F}_{d}$ into finite von Neumann algebras $\left(M_{k}, \tau_{k}\right)$ such that $\bigoplus \sigma_{k} \times \sigma_{k}^{\text {op }}$ is faithful. By considering $\tau=\sum 2^{-k} \tau_{k} \in T\left(\mathrm{C}^{*} \mathbb{F}_{d}\right)$, we are done.

We denote by $\mathbb{F}_{\infty}=\left\langle s_{1}, s_{2}, \ldots\right\rangle$ the free group on countably many generators. We identify the elements $s_{i}$ with the corresponding unitary elements in $\mathrm{C}^{*} \mathbb{F}_{\infty}$.

Theorem 29. Let $A$ be a $\mathrm{C}^{*}$-algebra having a tracial state $\tau$ and a dense sequence $u_{1}, u_{2}, \ldots$ of unitary elements in $A$. Let $\sigma: \mathrm{C}^{*} \mathbb{F}_{\infty} \rightarrow A, \sigma\left(s_{i}\right)=u_{i}$ be the corresponding *-homomorphism. Then, the finite von Neumann algebra $\pi_{\tau}(A)^{\prime \prime}$ satisfies CEC if and only if the linear functional defined by

$$
s_{i} \otimes s_{j} \mapsto \tau\left(u_{i} u_{j}^{*}\right) \quad \text { on } \quad \operatorname{span}\left\{s_{i} \otimes s_{j}\right\} \subset \mathrm{C}^{*} \mathbb{F}_{\infty} \otimes_{\min } \mathrm{C}^{*} \mathbb{F}_{\infty}
$$

is contractive.

In the operator space terminology, $\operatorname{span}\left\{s_{1}, s_{2}, \ldots\right\}$ is denoted by $\ell_{1}$, and the above theorem in particular says that $\mathrm{KC}$ holds true if the formal identity $\ell_{1} \otimes_{\min } \ell_{1} \hookrightarrow \mathrm{C}^{*} \mathbb{F}_{\infty} \otimes_{\max }$ $\mathrm{C}^{*} \mathbb{F}_{\infty}$ is contractive. It is known that this map is bounded (by $K_{G}^{\mathbb{C}}<1.41$ ). In case this map is moreover completely contractive, the theorem easily follows from Pisier's trick. See Section 12 in [Pi2] for more information.

Proof. We first prove the 'if' part. For notational simplicity, denote $C=\mathrm{C}^{*} \mathbb{F}_{\infty}$ and let it act on a Hilbert space $H$ with infinite multiplicity. We note that $C$ is $*$-isomorphic to its complex conjugate $\bar{C}$ acting on the conjugate Hilbert space $\bar{H}$ by $\bar{s}, s \in \mathbb{F}_{\infty}$. We use the standard identification of the Hilbert space $H \otimes \bar{H}$ with the space $S_{2}(H)$ of HilbertSchmidt class operators on $H$. Then, $a \otimes \bar{b} \in C \otimes \bar{C}$ acts on $S_{2}(H)$ by $(a \otimes \bar{b}) x=a x b^{*}$. By considering the complete isometries $s_{i} \mapsto s_{1}^{-1} s_{i}$, one may assume that $s_{1}=1$ and $\mathbb{F}_{\infty}=\left\langle s_{2}, s_{3}, \ldots\right\rangle$ in the above formula. Then, since every unital contraction is positive on a $\mathrm{C}^{*}$-algebra, the linear functional $s_{i} \otimes \bar{s}_{j} \mapsto \tau\left(u_{i} u_{j}^{*}\right)$ extends to a state $f$ on $C \otimes_{\min } \bar{C}$. Let $n \in \mathbb{N}$ be given and $\varepsilon=1 / n$. Then, there is $x \in S_{2}(H)$ of norm 1 such that

$$
\left|f\left(s_{i} \otimes \bar{s}_{j}\right)-\operatorname{Tr}\left(s_{i} x s_{j}^{*} x^{*}\right)\right|<\varepsilon^{2} / 8
$$

for $i, j \leq n$. Since $\left\|s_{i} x-x s_{i}\right\|_{2}^{2}=2\left(1-\Re \operatorname{Tr}\left(s_{i} x s_{i}^{*} x^{*}\right)\right)<(\varepsilon / 2)^{2}$, denoting $h=x^{*} x$, one has

$$
\left|\tau\left(u_{i} u_{j}^{*}\right)-\operatorname{Tr}\left(h s_{i} s_{j}^{*}\right)\right|<\varepsilon \text { and }\left\|s_{i} h s_{i}^{*}-h\right\|_{1}<\varepsilon
$$

for $i, j \leq n$. Here $\|\cdot\|_{1}$ denotes the trace norm. By approximation, one may assume that $h$ is of finite-rank and has rational eigenvalues. Then, Lemma 6.2.5 in [BO] implies that there is a u.c.p. map $\varphi_{n}$ from $C$ into the hyperfinite $\mathrm{II}_{1}$-factor $R$ such that $\tau\left(\varphi_{n}(a)\right)=\operatorname{Tr}(h a)$ for $a \in C$ and $\left|1-\tau\left(\varphi_{n}\left(s_{i}\right) \varphi_{n}\left(s_{i}\right)^{*}\right)\right| \leq 2 \varepsilon^{1 / 2}$ for $i \leq n$. See also Lemma 6.2.6 in [BO]. It follows that, for each $i$, the sequence $\left(\varphi_{n}\left(s_{i}\right)\right)_{n=1}^{\infty}$ gives rise to a unitary element $v_{i}$ in $R^{\omega}$ such that

$$
\tau\left(u_{i} u_{j}^{*}\right)=\tau\left(v_{i} v_{j}^{*}\right)
$$


for all $i, j$. By Theorem 27, $\pi_{\tau}(A)^{\prime \prime} \hookrightarrow R^{\omega}$.

For the converse implication, let $\pi_{\tau}(A)^{\prime \prime} \hookrightarrow R^{\omega}$ and $\left(u_{i}(n)\right)_{n=1}^{\infty} \in \prod R$ be liftings of $u_{i}$ in $R^{\omega}$. Then, for the corresponding $*$-homomorphism $\sigma_{n}: C \rightarrow R, s_{i} \mapsto u_{i}(n)$, the *-homomorphism $\sigma_{n} \times \sigma_{n}^{\text {op }}$ from $C \otimes C^{\text {op }}$ to $\mathbb{B}\left(L^{2}(R, \tau)\right)$ is continuous with respect to the minimal tensor product. Hence the map

$$
s_{i} \otimes s_{j} \mapsto \lim _{n}\left\langle\left(\sigma_{n} \times \sigma_{n}^{\mathrm{op}}\right)\left(s_{i} \otimes s_{j}\right) \hat{1}, \hat{1}\right\rangle=\lim _{n} \tau\left(u_{i}(n) u_{j}(n)^{*}\right)=\tau\left(u_{i} u_{j}^{*}\right)
$$

is contractive with respect to the minimal tensor norm.

Corollary 30 ([Ki1]). CEC is equivalent to $K C$ that $\mathrm{C}^{*} \mathbb{F}_{d} \otimes_{\max } \mathrm{C}^{*} \mathbb{F}_{d}=\mathrm{C}^{*} \mathbb{F}_{d} \otimes_{\min } \mathrm{C}^{*} \mathbb{F}_{d}$ holds for some/all $d \geq 2$.

Proof. Since the free groups $\mathbb{F}_{d}$ 's are embedded into each other (for $d \geq 2$ ), KC does not depends on $d \geq 2$. By Theorem 28, there is an embedding $\pi$ of $\mathrm{C}^{*} \mathbb{F}_{d}$ into a finite von Neumann algebra $M$ with separable predual (which may be assumed to be a factor by considering a free product) such that $\pi \times \pi^{\mathrm{op}}$ is faithful on $\mathrm{C}^{*} \mathbb{F}_{d} \otimes_{\max } \mathrm{C}^{*} \mathbb{F}_{d}$. If $M$ is embeddable into $R^{\omega}$, then one can lift the $*$-representation $\pi$ into $R^{\omega}$ to $\left(\pi_{n}\right)_{n=1}^{\infty}$ into $\prod R$ and sees that $\bigoplus \pi_{n} \times \pi_{n}^{\text {op }}$ is faithful. Since $R$ is hyperfinite, $\pi_{n} \times \pi_{n}^{\text {op }}$ factors through $\mathrm{C}^{*} \mathbb{F}_{d} \otimes_{\min } \mathrm{C}^{*} \mathbb{F}_{d}$ and $\mathrm{KC}$ follows. The converse implication follows from Theorem 29.

We remark that the free group $\mathbb{F}_{d}$ in $\mathrm{KC}$ can be replaced with any other single group which contains $\mathbb{F}_{2}$ and whose full group $\mathrm{C}^{*}$-algebra has the so-called local lifting property. In particular, $\mathrm{KC}$ is equivalent to the same statement but $\mathbb{F}_{d}$ is replaced with a "nontrivial" free product of amenable groups. See [BO, Ki1, Oz1] for the proof of these facts and more information.

16. Quasi-diagonality. In this section, we prove a weaker version of Kirchberg's Conjecture. We still assume $\mathbb{k}=\mathbb{C}$. Since $\mathrm{C}^{*} \mathbb{F}_{d}$ is $\mathrm{RFD}$, so is the minimal tensor product $\mathrm{C}^{*} \mathbb{F}_{d} \otimes_{\min } \mathrm{C}^{*} \mathbb{F}_{d}$. On the other hand, since any finite-dimensional $*$-representation of the maximal tensor product factors through the minimal tensor product, $\mathrm{KC}$ is equivalent to the assertion that $\mathrm{C}^{*}\left(\mathbb{F}_{d} \times \mathbb{F}_{d}\right)$ (which is canonically isomorphic to $\mathrm{C}^{*} \mathbb{F}_{d} \otimes_{\max } \mathrm{C}^{*} \mathbb{F}_{d}$ ) is RFD. By Positivstellensätze (Corollary 2), one obtains the following.

Corollary 31. $K C$ is equivalent to the following statement. If $f \in \mathbb{k}\left[\mathbb{F}_{d} \times \mathbb{F}_{d}\right]_{\mathrm{h}}$ is such that $\pi(f) \geq 0$ for all finite(-dimensional) unitary representations $\pi$ of $\mathbb{F}_{d} \times \mathbb{F}_{d}$, then $f \in \operatorname{arch}\left(\mathbb{k}\left[\mathbb{F}_{d} \times \mathbb{F}_{d}\right]_{+}\right)$.

While residual finite-dimensionality of $\mathrm{C}^{*}\left(\mathbb{F}_{d} \times \mathbb{F}_{d}\right)$ (Kirchberg's Conjecture) remains open, one can prove that it satisfies the following weaker property.

Definition. A $\mathrm{C}^{*}$-algebra $A$ is said to be quasi-diagonal if there is a net of u.c.p. maps $\varphi_{n}: A \rightarrow \mathbb{M}_{k(n)}(\mathbb{C})$ such that

$$
\left\|\varphi_{n}(x y)-\varphi_{n}(x) \varphi_{n}(y)\right\| \rightarrow 0
$$


for every $x, y \in A$, and

$$
\left\{x \in A: \varphi_{n}(x) \geq 0 \text { for all } n\right\}=A_{+} .
$$

Theorem $32([\mathrm{BO}])$. The $\mathrm{C}^{*}$-algebra $\mathrm{C}^{*}\left(\mathbb{F}_{d} \times \mathbb{F}_{d}\right)$ is quasi-diagonal.

The proof requires homotopy theory for $\mathrm{C}^{*}$-algebras. It is a celebrated theorem of Voiculescu that quasi-diagonality is a homotopy invariant. See Chapter 7 in [BO] for the proof of it and more information on quasi-diagonality. Recall that two $*$-homomorphisms $\pi_{0}, \pi_{1}$ from $A$ to $B$ are homotopic, denoted by $\pi_{0} \sim_{\mathrm{h}} \pi_{1}$, if they are connected by a pointwise continuous path $\left(\pi_{t}\right)_{t \in[0,1]}$ of $*$-homomorphisms; and $\mathrm{C}^{*}$-algebras $A$ and $B$ are homotopic if there are *-homomorphisms $\alpha: A \rightarrow B$ and $\beta: B \rightarrow A$ such that $\beta \circ \alpha \sim_{\mathrm{h}} \operatorname{id}_{A}$ and $\alpha \circ \beta \sim_{\mathrm{h}} \operatorname{id}_{B}$. We say $A$ is homotopically contained in $B$ if there are a $\mathrm{C}^{*}$-algebra $B_{1}$ which is homotopic to $B$, a $*$-homomorphism $\alpha: A \rightarrow B_{1}$, and a u.c.p. map $\beta: B_{1} \rightarrow A^{* *}$ such that $\beta \circ \alpha=\iota_{A}$, the canonical embedding of $A$ into $A^{* *}$. We note that if there are $*$-homomorphisms $\alpha: A \rightarrow B$ and $\beta: B \rightarrow A^{* *}$ such that $\beta \circ \alpha \sim_{\mathrm{h}} \iota_{A}$, then $A$ is homotopically contained in $B$. Indeed, if $\gamma_{t}$ is a homotopy from $\iota_{A}$ to $\beta \circ \alpha$, then $\gamma \oplus \alpha$ gives rise to an embedding of $A$ into the mapping cylinder

$$
Z_{\beta}=\left\{(f, b) \in C\left([0,1], A^{* *}\right) \oplus B: f(1)=\beta(b)\right\},
$$

which is homotopic to $B$.

Proof of Theorem 32. This follows from Voiculescu's theorem, once we observe that the property being homotopically contained in the trivial $\mathrm{C}^{*}$-algebra $\mathbb{C} 1$ (or finite-dimensional $\mathrm{C}^{*}$-algebras, see [Oz2]) is preserved by unital full free products and tensor products. Note that $\mathrm{C}^{*} \mathbb{Z} \cong C(\mathbb{T}) \subset C([0,1])$ has this property, to begin with.

It is unclear for which group $\Gamma$, the full group $\mathrm{C}^{*}$-algebra $\mathrm{C}^{*} \Gamma$ is quasi-diagonal (or homotopically contained in $\mathbb{C} 1$ - this is not the case when $\mathrm{C}^{*} \Gamma$ has nontrivial projections, e.g., when $\Gamma$ has torsion or Kazhdan's property $(\mathrm{T})$ ). A well-known conjecture of Rosenberg (see $[\mathrm{C}+]$ ) asserts that all amenable groups should have quasi-diagonal $\mathrm{C}^{*} \Gamma$. On the other extreme, any infinite simple Kazhdan's property (T) group gives rise to a counterexample $([\mathrm{Ki} 2])$. The case for $\mathrm{SL}_{3}(\mathbb{Z})$ is unclear (cf. [Be1, Be2]).

17. Operator systems. We will prove the Choi-Effros theorem giving the abstract characterization of semi-operator systems. Recall that a (semi-)operator system is a unital *-subspace $\mathcal{S}$ of a (semi-pre-) $\mathrm{C}^{*}$-algebra $\mathcal{A}$, equipped with the matricial positive cone $\mathbb{M}_{n}(\mathcal{S})_{+}:=\mathbb{M}_{n}(\mathcal{S}) \cap \mathbb{M}_{n}(\mathcal{A})_{+}$for each $n \in \mathbb{N}$. Here, we do not assume it norm-closed. We generally think that the specific embedding $\mathcal{S} \subset \mathcal{A}$ is not a part of the operator system structure of $\mathcal{S}$, but only the matricial positive cones $\left(\mathbb{M}_{n}(\mathcal{S})_{+}\right)_{n=1}^{\infty}$ are. A map $\varphi: \mathcal{S} \rightarrow \mathcal{T}$ between operator systems is said to be completely positive (c.p.) if id $\otimes \varphi: \mathbb{M}_{n}(\mathcal{S}) \rightarrow \mathbb{M}_{n}(\mathcal{T})$ is positive for all $n$. Thus $\mathcal{S}$ and $\mathcal{T}$ are completely order isomorphic if there is a unital c.p. (u.c.p.) linear isomorphism $\varphi: \mathcal{S} \rightarrow \mathcal{T}$ such that $\varphi^{-1}$ is 
also c.p. It is not too hard to see that a semi-operator system $\left(\mathcal{S},\left(\mathbb{M}_{n}(\mathcal{S})_{+}\right)_{n=1}^{\infty}\right)$ satisfies the following axiom:

(i) $\mathbb{R}_{\geq 0} 1 \in \mathbb{M}_{n}(\mathcal{S})_{+}$and $\lambda a+b \in \mathbb{M}_{n}(\mathcal{S})_{+}$for every $a, b \in \mathbb{M}_{n}(\mathcal{S})_{+}$and $\lambda \in \mathbb{R}_{\geq 0}$;

(ii) $x^{*} a x \in \mathbb{M}_{n}(\mathcal{S})_{+}$for every $m, n \in \mathbb{N}, a \in \mathbb{M}_{m}(\mathcal{S})_{+}$and $x \in \mathbb{M}_{m, n}(\mathbb{k})$;

(iii) For every $n$ and every $h \in \mathbb{M}_{n}(\mathcal{S})_{\mathrm{h}}$, there is $R>0$ such that $h \leq R 1$.

If $\mathcal{S}$ is an operator system, then it moreover satisfies the following:

(iv) $\mathbb{M}_{n}(\mathcal{S})_{+} \cap\left(-\mathbb{M}_{n}(\mathcal{S})_{+}\right)=\{0\}$ and $\mathbb{M}_{n}(\mathcal{S})_{+}$is archimedean closed;

$(\mathrm{v}) I(\mathcal{S}):=\left\{x \in \mathcal{S}:\left[\begin{array}{cc}0 & x^{*} \\ x & 0\end{array}\right] \in \operatorname{arch}\left(\mathbb{M}_{2}(\mathcal{S})_{+}\right)\right\}=\{0\}$.

We recall the ground assumption that the unit is always written as 1 , and so is the unit matrix in $\mathbb{M}_{n}(\mathbb{k})$. The definition of $x^{*} a x$ is the obvious one:

$$
x^{*} a x=\left[\sum_{k, l} x_{k, i}^{*} a_{k, l} x_{l, j}\right]_{i, j}=\left[\sum_{k, l} x_{k, i}^{*} x_{l, j} a_{k, l}\right]_{i, j} \text { for } x=\left[x_{k, i}\right]_{k, i} \text { and } a=\left[a_{k, l}\right]_{k, l} .
$$

An abstract (semi-)operator system is a system $\left(\mathcal{S},\left(\mathbb{M}_{n}(\mathcal{S})_{+}\right)_{n=1}^{\infty}\right)$ satisfying the above axiom. The condition (v) follows from other conditions in case $\mathbb{k}=\mathbb{C}$. Given an abstract semi-operator system $\mathcal{S}$, we introduce the corresponding semi-pre-C $\mathrm{C}^{*}$-algebra $\mathcal{A}(\mathcal{S})$ as the universal unital $*$-algebra generated by $\mathcal{S}$, equipped with the $*$-positive cone

$$
\mathcal{A}(\mathcal{S})_{+}=\left\{x^{*} a x: n \in \mathbb{N}, a \in \mathbb{M}_{n}(\mathcal{S})_{+}, x \in \mathbb{M}_{n, 1}(\mathcal{A}(\mathcal{S}))\right\} .
$$

To see that the Combes axiom is satisfied, let $x \in \mathcal{S}$ be given arbitrary. Then, there is $R>0$ such that $\left[\begin{array}{cc}R 1 & -x \\ -x^{*} & R 1\end{array}\right] \in \mathbb{M}_{2}(\mathcal{S})_{+}$. It follows that

$$
0 \leq \frac{1}{R}\left[\begin{array}{ll}
x^{*} & R 1
\end{array}\right]\left[\begin{array}{cc}
R 1 & -x \\
-x^{*} & R 1
\end{array}\right]\left[\begin{array}{c}
x \\
R 1
\end{array}\right]=R^{2} 1-x^{*} x .
$$

This implies that $\mathcal{S} \subset \mathcal{A}(\mathcal{S})^{\text {bdd }}$ and hence the Combes axiom follows. The universal $\mathrm{C}^{*}$ algebra $\mathrm{C}_{\mathrm{u}}^{*}(\mathcal{A}(\mathcal{S}))$ of $\mathcal{A}(\mathcal{S})$ is generated by $\iota(\mathcal{S})$ and has the following universal property: Every u.c.p. map $\mathcal{S}$ into a $\mathrm{C}^{*}$-algebra $B$ extends to a $*$-homomorphism on $\mathrm{C}_{\mathrm{u}}^{*}(\mathcal{A}(\mathcal{S})$ ). We call $\mathrm{C}_{\mathrm{u}}^{*}(\mathcal{A}(\mathcal{S}))$ the universal $\mathrm{C}^{*}$-algebra of the semi-operator system $\mathcal{S}$ and denote it simply by $\mathrm{C}_{\mathrm{u}}^{*}(\mathcal{S})$.

Theorem $33([\mathrm{CE}])$. Let $\mathcal{S}$ be a semi-operator system. Then, one has

$$
\operatorname{ker}\left(\iota: \mathcal{S} \rightarrow \mathrm{C}_{\mathrm{u}}^{*}(\mathcal{S})\right)=I(\mathcal{S})
$$

and

$$
\operatorname{arch}\left(\mathbb{M}_{n}(\mathcal{S})_{+}\right)=\mathbb{M}_{n}(\mathcal{S})_{\mathrm{h}} \cap \iota^{-1}\left(\mathbb{M}_{n}\left(\mathrm{C}_{\mathrm{u}}^{*}(\mathcal{S})\right)_{+}\right)
$$

for every $n$. In particular, an abstract operator system is completely order isomorphic to a concrete operator system.

Proof. By definition, one has $\mathbb{M}_{n}(\mathcal{S})_{+} \subset \mathbb{M}_{n}\left(\mathrm{C}_{\mathrm{u}}^{*}(\mathcal{S})\right)_{+}$. Also, it is not difficult to see $I(\mathcal{S}) \subset$ ker $\iota$. For the converse inclusions, it suffices to show that for every $a \in \mathbb{M}_{n}(\mathcal{S})_{\mathrm{h}} \backslash$ $\operatorname{arch}\left(\mathbb{M}_{n}(\mathcal{S})_{+}\right)$, there is a positive $*$-representation $\pi$ on $\mathrm{C}_{\mathrm{u}}^{*}(\mathcal{S})$ such that $(\mathrm{id} \otimes \pi)(a) \nsupseteq 0$. (This in particular shows that $\operatorname{ker} \iota \subset I(\mathcal{S})$.) For this, it is enough to find a u.c.p. 
map $\psi: \mathcal{S} \rightarrow \mathbb{B}(H)$ such that $(\mathrm{id} \otimes \psi)(a) \nsupseteq 0$. Since $\mathbb{M}_{n}(\mathcal{S})_{+}$is algebraically solid in $\mathbb{M}_{n}(\mathcal{S})_{\mathrm{h}}$, one may find a state $\tilde{\varphi}$ on $\mathbb{M}_{n}(\mathcal{S})$ such that $\tilde{\varphi}(a)<0$. Let $\varphi: \mathcal{S} \rightarrow \mathbb{M}_{n}\left(\mathbb{k}_{k}\right)$ be the corresponding c.p. map to $\tilde{\varphi}$ (see Lemma 16). Then, $\varphi(1) \in \mathbb{M}_{n}(\mathbb{k})$ is a self-adjoint positive element and for the support projection $p$ of $\varphi(1)$, one has $\varphi(x)=p \varphi(x) p$ for all $x \in \mathcal{S}$ (because $\left[\begin{array}{cc}R \varphi(1) & \varphi(x)^{*} \\ \varphi(x) & R \varphi(1)\end{array}\right] \geq 0$ for some $\left.R>0\right)$. It follows that the map $\psi$ defined by $\psi(x)=\left.\varphi(1)^{-1 / 2} \varphi(x) \varphi(1)^{-1 / 2}\right|_{p \ell_{2}^{n}}$ is a u.c.p. map from $\mathcal{S}$ into $\mathbb{B}\left(p \ell_{2}^{n}\right)$ such that $\psi(a) \nsupseteq 0$. This completes the proof.

The proof above is short and classical, but it would be nicer if there is another proof which relies more directly on the definition of $\mathcal{A}(\mathcal{S})_{+}$. There is another very interesting $\mathrm{C}^{*}$-algebra associated with an operator system $\mathcal{S}$, called the $\mathrm{C}^{*}$-envelope $\mathrm{C}_{\mathrm{e}}^{*}(\mathcal{S})$ of $\mathcal{S}$ (see Chapter 15 in $[\mathrm{Pa}])$, but the explicit description of the corresponding semi-pre- $\mathrm{C}^{*}$-algebra structure on $\mathcal{A}(\mathcal{S})$ is unclear.

18. Operator system duality and tensor products. An important corollary to Theorem 33 is the operator system duality. Let $\mathcal{S}$ be a semi-operator system. We denote by $\mathcal{S}^{\text {d }}$ the linear dual of $\mathcal{S}$, and identify $\mathbb{M}_{n}\left(\mathcal{S}^{\text {d }}\right)$ with the space of the linear maps from $\mathcal{S}$ to $\mathbb{M}_{n}(\mathbb{k})$ (cf. Lemma 16) to introduce the corresponding matricial positive cones on it. Thus for $f=\left[f_{i, j}\right]_{i, j} \in \mathbb{M}_{n}\left(\mathcal{S}^{\mathrm{d}}\right)$, one has $f^{*}=\left[f_{j, i}^{*}\right]_{i, j}$ (recall that $\left.f^{*}(x)=f\left(x^{*}\right)^{*}\right)$, and $f \geq 0$ in $\mathbb{M}_{n}\left(\mathcal{S}^{\mathrm{d}}\right)$ if and only if $x \mapsto\left[f_{i, j}(x)\right] \in \mathbb{M}_{n}(\mathbb{k})$ is c.p. Then, the system $\left(\mathcal{S}^{\mathrm{d}},\left(\mathbb{M}_{n}\left(\mathcal{S}^{\mathrm{d}}\right)_{+}\right)_{n=1}^{\infty}\right)$ satisfies all the axioms of operator systems in Section 17, except the ones involving the unit 1. We also observe by Theorem 33 (and Theorem 14) that $a \in \mathbb{M}_{m}(\mathcal{S})_{\mathrm{h}}$ belongs to $\operatorname{arch}\left(\mathbb{M}_{m}(\mathcal{S})_{+}\right)$if and only if $a$ is c.p. as a linear map from $\mathcal{S}^{\text {d }}$ to $\mathbb{M}_{m}(\mathbb{k})$. It follows that $\mathcal{S} \hookrightarrow \mathcal{S}^{\text {dd }}$ is a complete order isomorphic embedding modulo archimedean closure. When $\mathcal{S}$ is a finite-dimensional operator system, then one can pick any faithful state $p$ on $\mathcal{S}$, and make $\left(\mathcal{S}^{\mathrm{d}},\left(\mathbb{M}_{n}\left(\mathcal{S}^{\mathrm{d}}\right)_{+}\right)_{n=1}^{\infty}\right)$ an abstract operator system with the unit $p$. (So, the operator system structure of $\mathcal{S}^{\text {d }}$ depends on the choice of the unit $p$, and one has to choose the canonical one if exists. For example, the unit of $\mathcal{S}^{\mathrm{dd}}=\left(\mathcal{S}^{\mathrm{d}}\right)^{\mathrm{d}}$ has to be the unit 1 of $\mathcal{S}$.) Summarizing the above discussion, we reach to the following corollary.

Corollary $34([\mathrm{CE}])$. Let $\mathcal{S}$ be a finite-dimensional operator system. Then, its dual $\mathcal{S}^{\mathrm{d}}$ is an (abstract) operator system. Moreover, the natural isomorphism $\mathcal{S}=\mathcal{S}^{\mathrm{dd}}$ is a complete order isomorphism.

Let $\mathcal{S}$ and $\mathcal{T}$ be (semi-)operator systems, and $\mathcal{S} \otimes \mathcal{T}$ be their tensor product. There are two (in fact more) canonical operator system structures on $\mathcal{S} \otimes \mathcal{T}([\mathrm{K}+, \mathrm{FP}])$. The first one is the minimal tensor product $\mathcal{S} \otimes^{\min } \mathcal{T}$, defined by

$$
\mathbb{M}_{n}\left(\mathcal{S} \otimes{ }^{\min } \mathcal{T}\right)_{+}=\mathbb{M}_{n}(\mathcal{S} \otimes \mathcal{T}) \cap\left(\mathrm{id} \otimes \iota_{\mathcal{S}} \otimes \iota_{\mathcal{T}}\right)^{-1}\left(\mathbb{M}_{n}\left(\mathrm{C}_{\mathrm{u}}^{*}(\mathcal{S}) \otimes{ }^{\min } \mathrm{C}_{\mathrm{u}}^{*}(\mathcal{T})\right)_{+}\right) .
$$

We observe from Theorem 14 that an element $c \in \mathbb{M}_{n}(\mathcal{S} \otimes \mathcal{T})_{\mathrm{h}}$ is positive if and only if $(\mathrm{id} \otimes \varphi \otimes \psi)(c) \in \mathbb{M}_{n k l}(\mathbb{k})_{+}$for all $k, l \in \mathbb{N}$ and c.p. maps $\varphi: \mathcal{S} \rightarrow \mathbb{M}_{k}(\mathbb{k})$ and $\psi: \mathcal{T} \rightarrow$ 
$\mathbb{M}_{l}(\mathbb{k})$. The second one is the maximal operator system tensor product $\mathcal{S} \otimes^{\max } \mathcal{T}$, defined by

$$
\mathbb{M}_{n}\left(\mathcal{S} \otimes{ }^{\max } \mathcal{T}\right)_{+}=\operatorname{arch}\left(\left\{x^{*}(s \otimes t) x: x \in \mathbb{M}_{k l, n}(\mathbb{k}), s \in \mathbb{M}_{k}(\mathcal{S})_{+}, t \in \mathbb{M}_{l}(\mathcal{T})_{+}\right\}\right),
$$

where $x^{*}(s \otimes t) x=\left[\sum_{p, p^{\prime}, q, q^{\prime}} x_{(p, q), i}^{*}\left(s_{p, p^{\prime}} \otimes t_{q, q^{\prime}}\right) x_{\left(p^{\prime}, q^{\prime}\right), j}\right]_{i, j} \in \mathbb{M}_{n}(\mathcal{S} \otimes \mathcal{T})$. We note that for $\mathcal{S}_{1} \subset \mathcal{S}_{2}$ and $\mathcal{T}_{1} \subset \mathcal{T}_{2}$, the natural embedding $\mathcal{S}_{1} \otimes \mathcal{T}_{1} \hookrightarrow \mathcal{S}_{2} \otimes \mathcal{T}_{2}$ is a complete order isomorphic embedding with respect to minimal tensor products, but need not be so with respect to the maximal tensor products. It is not too hard to see that for any operator systems $\mathcal{S}$ and $\mathcal{T}$, both minimal and maximal tensor product satisfy the axiom of operator systems, and that the formal identity map from $\mathcal{S} \otimes^{\max } \mathcal{T}$ to $\mathcal{S} \otimes^{\min } \mathcal{T}$ is c.p. These two tensor products are dual to each other in the following sense.

Theorem $35([\mathrm{FP}])$. Let $\mathcal{S}$ and $\mathcal{T}$ be finite-dimensional operator systems. Then the natural isomorphism

$$
\left(\mathcal{S} \otimes^{\min } \mathcal{T}\right)^{\mathrm{d}}=\mathcal{S}^{\mathrm{d}} \otimes^{\max } \mathcal{T}^{\mathrm{d}}
$$

is a complete order isomorphism.

Proof. First, we observe that if $p$ and $q$ are faithful states, then $p \otimes q$ is faithful on $\mathcal{S} \otimes{ }^{\min } \mathcal{T}$. We will prove this for general operator systems. Let $(p \otimes q)(c)=0$ for some $c \in\left(\mathcal{S} \otimes \otimes^{\min } \mathcal{T}\right)_{+}$. Then, the faithfulness of $p$ implies $(\mathrm{id} \times q)(c)=0$ in $\mathcal{S}$. Thus, for every c.p. $\operatorname{map} \varphi: \mathcal{S} \rightarrow \mathbb{M}_{m}(\mathbb{k})$, one has $(\mathrm{id} \otimes q)((\varphi \otimes \mathrm{id})(c))=\varphi((\mathrm{id} \times q)(c))=0$. Since id $\otimes q$ is also faithful on $\mathbb{M}_{m}(\mathcal{T})$, this implies $(\varphi \otimes \mathrm{id})(c)=0$, and $c=0$.

By replacing $\mathcal{S}$ and $\mathcal{T}$ with their duals, it suffices to show that the natural map $\mathcal{S}^{\mathrm{d}} \otimes^{\text {min }}$ $\mathcal{T}^{\mathrm{d}} \rightarrow\left(\mathcal{S} \otimes{ }^{\max } \mathcal{T}\right)^{\mathrm{d}}$ is a complete order isomorphism. For complete positivity, one has to show for any $f \in \mathbb{M}_{n}\left(\mathcal{S}^{\mathrm{d}} \otimes^{\min } \mathcal{T}^{\mathrm{d}}\right)_{+}$, the corresponding map $\tilde{f}: \mathcal{S} \otimes^{\max } \mathcal{T} \rightarrow \mathbb{M}_{n}(\mathbb{k})$ is c.p. But this is true, since for every $m, k, l$ and $x \in \mathbb{M}_{k l, m}(\mathbb{k}), s \in \mathbb{M}_{k}(\mathcal{S})_{+}$, and $t \in \mathbb{M}_{l}(\mathcal{T})_{+}$, one has

$$
\tilde{f}\left(x^{*}(s \otimes t) x\right)=x^{*}\langle f, s \otimes t\rangle x \in \mathbb{M}_{m n}(\mathbb{k})_{+},
$$

thanks to the completely order isomorphic embeddings $\mathcal{S} \subset \mathcal{S}^{\text {dd }}$ and $\mathcal{T} \subset \mathcal{T}^{\text {dd }}$. It remains to show that if $c:\left(\mathcal{S} \otimes^{\max } \mathcal{T}\right)^{\mathrm{d}} \rightarrow \mathbb{M}_{n}(\mathbb{k})$ is c.p., then it is c.p. on $\mathcal{S}^{\mathrm{d}} \otimes^{\min } \mathcal{T}^{\mathrm{d}}$. But by definition, $c$ is identified with an element in $\mathbb{M}_{n}\left(\left(\mathcal{S} \otimes^{\max } \mathcal{T}\right)^{\mathrm{dd}}\right)_{+}=\mathbb{M}_{n}\left(\mathcal{S} \otimes^{\max } \mathcal{T}\right)_{+}$and $c+\varepsilon 1=x^{*}(s \otimes t) x$ for some $x, s, t$. Hence, for every $f \in \mathbb{M}_{m}\left(\mathcal{S}^{\mathrm{d}} \otimes \otimes^{\min } \mathcal{T}^{\mathrm{d}}\right)_{+}$, one has

$$
(\mathrm{id} \otimes(c+\varepsilon 1))(f)=x^{*}\langle s \otimes t, f\rangle x \in \mathbb{M}_{n m}(\mathbb{k})_{+} .
$$

Since $\varepsilon>0$ was arbitrary, one has $(\mathrm{id} \otimes c)(f) \in \mathbb{M}_{n m}(\mathbb{k})_{+}$. This completes the proof.

19. Quantum correlation matrices and Tsirelson's Problem. In this section, we take $\mathbb{k}=\mathbb{C}$. In quantum information theory, a measurement of a state is carried out by so-called POVMs (positive operator valued measures). For simplicity of the presentation, we only consider PVMs (projection valued measures). A PVM with $m$ outputs is an $m$-tuple $\left(P_{i}\right)_{i=1}^{m}$ of orthogonal projections on a Hilbert space $H$ such that $\sum P_{i}=1$. For 
each $n \in \mathbb{N}$, the convex sets of quantum correlation matrices of two separated systems of $d$ PVMs with $m$-outputs are defined by

$$
\mathcal{Q}_{c}^{n}=\left\{\left[V^{*} P_{i}^{(k)} Q_{j}^{(l)} V\right]_{k, l}: \begin{array}{c}
H \text { a Hilbert space, } V: \ell_{2}^{n} \rightarrow H \text { an isometry } \\
\left(P_{i}^{(k)}\right)_{i=1}^{m}, k=1, \ldots, d, \text { PVMs on } H, \\
\left(Q_{j}^{(l)}\right)_{j=1}^{m}, l=1, \ldots, d, \text { PVMs on } H, \\
{\left[P_{i}^{(k)}, Q_{j}^{(l)}\right]=0 \text { for all } i, j \text { and } k, l}
\end{array}\right.
$$

(here $[A, B]=A B-B A$ is the commutator) and

$$
\mathcal{Q}_{s}^{n}=\operatorname{closure}\left\{\left[V^{*}\left(P_{i}^{(k)} \otimes Q_{j}^{(l)}\right) V\right]_{k, l}: \begin{array}{c}
V: \ell_{2}^{n} \rightarrow H \otimes K \text { an isometry, } \\
\left(P_{i}^{(k)}\right)_{i=1}^{m}, k=1, \ldots, d, \text { PVMs on } H, \\
\left(Q_{j}^{(l)}\right)_{j=1}^{m}, l=1, \ldots, d, \text { PVMs on } K
\end{array}\right\} .
$$

The sets $\mathcal{Q}_{c}^{n}$ and $\mathcal{Q}_{s}^{n}$ are closed convex subsets of $\mathbb{M}_{m d}\left(\mathbb{M}_{n}(\mathbb{C})_{+}\right)$such that $\mathcal{Q}_{s}^{n} \subset \mathcal{Q}_{c}^{n}$. We simply write $\mathcal{Q}_{c}$ and $\mathcal{Q}_{s}$ when $n=1$. Whether $\mathcal{Q}_{c}=\mathcal{Q}_{s}$ for every $m$ and $d$ is the well-known Tsirelson Problem. We refer the reader to [Fr, J+, Ts] for the background and the literature on this problem. (The "real" problem may be that which of $\mathcal{Q}_{c}$ and $\mathcal{Q}_{s}$ should we take as the definition of quantum correlation matrices? See [Fr, Oz2].) Since a commuting system on a finite-dimensional Hilbert space splits as a tensor product if irreducible, one also has

$$
\mathcal{Q}_{s}^{n}=\operatorname{closure}\left\{\left[V^{*} P_{i}^{(k)} Q_{j}^{(l)} V\right]_{k, l}: \begin{array}{c}
\operatorname{dim} H<\infty, V: \ell_{2}^{n} \rightarrow H \text { an isometry } \\
\left(P_{i}^{(k)}\right)_{i=1}^{m}, k=1, \ldots, d, \text { PVMs on } H, \\
\left(Q_{j}^{(l)}\right)_{j=1}^{m}, l=1, \ldots, d, \text { PVMs on } H, \\
{\left[P_{i}^{(k)}, Q_{j}^{(l)}\right]=0 \text { for all } i, j \text { and } k, l}
\end{array}\right\},
$$

which looks more similar to $\mathcal{Q}_{c}^{n}$. As observed in [Fr, J+], Tsirelson's Problem is a problem about the semi-pre-C*-algebra

$$
\mathfrak{F}_{m}^{d}=\ell_{\infty}^{m} * \cdots * \ell_{\infty}^{m}
$$

the $d$-fold unital free product of $\ell_{\infty}^{m}$ (see Example 5 ). This algebra is also $*$-isomorphic to the group algebra $\mathbb{C}[\Gamma]$ of $\Gamma=\mathbb{Z}_{m}^{* d}$, the $d$-fold free product of the finite cyclic group $\mathbb{Z}_{m}$. We denote by $\left(p_{i}\right)_{i=1}^{m}$ the standard basis of minimal projections in $\ell_{\infty}^{m}$, and by $\left(p_{i}^{(k)}\right)_{i=1}^{m}$ the $k$-th copy of it in the free product $\mathfrak{F}_{m}^{d}$. We also write $p_{i}^{(k)}$ for the elements $p_{i}^{(k)} \otimes 1$ in $\mathfrak{F}_{m}^{d} \otimes \mathfrak{F}_{m}^{d}$ and $q_{j}^{(l)}$ for $1 \otimes p_{j}^{(l)}$. Now, it is not too hard to see that

$$
\mathcal{Q}_{c}^{n}=\left\{\left[\varphi\left(p_{i}^{(k)} q_{j}^{(l)}\right)\right]_{k, l}: \varphi \in S_{n}\left(\mathfrak{F}_{m}^{d} \otimes^{\max } \mathfrak{F}_{m}^{d}\right)\right\}
$$

and

$$
\mathcal{Q}_{s}^{n}=\left\{\left[\varphi\left(p_{i}^{(k)} q_{j}^{(l)}\right)\right]_{i, l}: \varphi \in S_{n}\left(\mathfrak{F}_{m}^{d} \otimes^{\min } \mathfrak{F}_{m}^{d}\right)\right\}
$$


Here we recall that $S_{n}(\mathcal{A})$ denotes the set of u.c.p. maps from $\mathcal{A}$ into $\mathbb{M}_{n}(\mathbb{C})$. When $(m, d)=(2,2)$, the group $\mathbb{Z}_{2}^{* 2}$ is isomorphic to the infinite dihedral group $D_{\infty}$ and the universal representation $\left\langle\left[\begin{array}{ll}0 & 1 \\ 1 & 0\end{array}\right],\left[\begin{array}{cc}0 & z \\ z^{*} & 0\end{array}\right]\right\rangle \subset \mathbb{M}_{2}(C(\mathbb{T}))$ gives rise to an embedding $\mathrm{C}^{*} D_{\infty} \subset$ $\mathbb{M}_{2}(C(\mathbb{T}))$. In particular, $\Gamma$ is amenable and $\mathcal{Q}_{c}^{n}=\mathcal{Q}_{s}^{n}$ in this case. Otherwise, $\mathbb{Z}_{m}^{* d}$ is a "nontrivial" free product group and contains a copy of $\mathbb{F}_{2}$. It is proved in $[\mathrm{Fr}, \mathrm{J}+]$ that the matricial version of Tsirelson's Problem (conjectures (2) and (3) in the following theorem) is equivalent to $\mathrm{CEC}$ and $\mathrm{KC}$. Here we prove that the original Tsirelson Problem is also equivalent to them.

Theorem 36 (cf. [Fr, J+]). The following conjectures are equivalent.

(1) The Connes Embedding Conjecture holds true.

(2) One has $\mathcal{Q}_{c}^{n}=\mathcal{Q}_{s}^{n}$ for all $m, d, n \in \mathbb{N}$.

(3) There are $m, d \geq 2$ with $(m, d) \neq(2,2)$ such that $\mathcal{Q}_{c}^{n}=\mathcal{Q}_{s}^{n}$ for all $n$.

(4) One has $\mathcal{Q}_{c}=\mathcal{Q}_{s}$ for all $m, d \in \mathbb{N}$.

Proof. The implication $(1) \Rightarrow(2)$ follows from the fact that $\mathrm{KC}$ is equivalent to that $\mathfrak{F}_{m}^{d} \otimes^{\max } \mathfrak{F}_{m}^{d}=\mathfrak{F}_{m}^{d} \otimes^{\min } \mathfrak{F}_{m}^{d}$ (see Section 15). That $(2) \Rightarrow(3) \&(4)$ is obvious. Now, if (3) holds true, then the operator system structures on

$$
\operatorname{span}\left\{p_{i}^{(k)} q_{j}^{(l)}: i, j, k, l\right\}=\left(\ell_{\infty}^{m}+\cdots+\ell_{\infty}^{m}\right) \otimes\left(\ell_{\infty}^{m}+\cdots+\ell_{\infty}^{m}\right) \subset \mathfrak{F}_{m}^{d} \otimes \mathfrak{F}_{m}^{d}
$$

induced from max and min tensor products coincide by Corollary 34. This implies KC by Corollary 20 . This proves $(3) \Rightarrow(1)$. Finally, it remains to show $(4) \Rightarrow(1)$. Let a finite von Neumann algebra $(M, \tau)$ and a sequence $u_{1}, u_{2}, \ldots$ of unitary elements be given. By spectral theorem, for every $m$, there are unitary elements $u_{i}(m)$ of order $m$ such that $\left\|u_{i}-u_{i}(m)\right\| \leq \pi / m$. Recall that $\mathfrak{F}_{m}^{d} \cong \mathbb{C}\left[\mathbb{Z}_{m}^{* d}\right]$ via $\sum_{i=1}^{m} \omega_{m}^{i} p_{i}^{(k)} \leftrightarrow s_{k}$, where $\omega_{m}$ is an $n$-th primitive root of unity and $s_{k}$ is the generator of the $k$-th copy of $\mathbb{Z}_{m}$ in $\mathbb{Z}_{m}^{* d}$. Then, condition (4) for $m, d$ implies that the map

$$
s_{i} \otimes s_{j} \mapsto \tau\left(u_{i}(m) u_{j}(m)^{*}\right)=\left\langle\pi_{\tau}\left(u_{i}(m)\right) \pi_{\tau}^{\mathrm{op}}\left(\left(u_{j}(m)^{*}\right)^{\mathrm{op}}\right) \hat{1}, \hat{1}\right\rangle
$$

is contractive on $\operatorname{span}\left\{s_{i} \otimes s_{j}: i, j \leq d\right\} \subset \mathrm{C}^{*} \mathbb{Z}_{m}^{* d} \otimes_{\min } \mathrm{C}^{*} \mathbb{Z}_{m}^{* d}$. It is also contractive on $\mathrm{C}^{*} \mathbb{F}_{d} \otimes_{\text {min }} \mathrm{C}^{*} \mathbb{F}_{d}$ through the natural surjection $\mathbb{F}_{d} \rightarrow \mathbb{Z}_{m}^{* d}$. Taking limit in $m$ and $d$, one verifies that the condition in Theorem 29 holds. It follows that $(M, \tau)$ satisfies CEC. (Playing around with the fact that every element in a $\mathrm{II}_{1}$-factor is a linear combination of projections with control on the number of terms and coefficients $([\mathrm{FH}])$, one can weaken condition (4) further to the extent that $\mathcal{Q}_{c}=\mathcal{Q}_{s}$ for a fixed $m \geq 2$ and all $d \in \mathbb{N}$.)

20. Quantum correlation matrices and semidefinite program. We continue the study of Tsirelson's Problem whether $\mathcal{Q}_{c}=\mathcal{Q}_{s}$. We will see that $\mathcal{Q}_{s} \subset \mathcal{Q}_{c}$ are approximated from above and from below by rather explicit semi-algebraic sets. Recall that a subset of $\mathbb{R}^{N}$ is said to be semi-algebraic if it can be defined by finitely many polynomial equations and inequalities. It is very likely that $\mathcal{Q}_{c}$ and $\mathcal{Q}_{s}$ themselves are not semialgebraic (cf. Problem 2.10 in [Ts]), except the case $(m, d)=(2,2)$ where $\mathcal{Q}_{c}=\mathcal{Q}_{s}$ is 
semi-algebraic (since the irreducible representations of $\mathbb{Z}_{2}^{* 2}$ are at most two dimensional). For the description of $\mathcal{Q}_{c}$, it is probably neater to use the isomorphism $\ell_{\infty}^{m} \cong \mathbb{C}\left[\mathbb{Z}_{m}\right]$, given by the Fourier transform $p_{i} \leftrightarrow \frac{1}{m} \sum_{v=1}^{m} \omega_{m}^{-i v} s^{v}$, where $\omega_{m}$ is an $n$-th primitive root of unity and $s$ is the generator of $\mathbb{Z}_{m}$. Now, $\mathfrak{F}_{m}^{d} \otimes^{\max } \mathfrak{F}_{m}^{d}$ is identified with $\mathbb{C}\left[\mathbb{Z}_{m}^{* d} \times \mathbb{Z}_{m}^{* d}\right]$. We write $s_{k}$ and $t_{l}$ for the generator of $\mathbb{Z}_{m}^{(k)} \times\{1\}$ and $\{1\} \times \mathbb{Z}_{m}^{(l)}$ in $\mathbb{Z}_{m}^{* d} \times \mathbb{Z}_{m}^{* d}$. By Corollary 13, there is a one-to-one correspondence between a positive type function $f$ on $\mathbb{Z}_{m}^{* d} \times \mathbb{Z}_{m}^{* d}$ and a state $\varphi_{f}$ on $\mathbb{C}\left[\mathbb{Z}_{m}^{* d} \times \mathbb{Z}_{m}^{* d}\right]$, given by $\varphi_{f}(g)=\sum_{t} f(t) g(t)$. Let $E_{n}$ be an increasing and exhausting sequence of finite subsets of $\mathbb{Z}_{m}^{* d} \times \mathbb{Z}_{m}^{* d}$. We say a function $f: E_{n}^{-1} E_{n} \rightarrow \mathbb{C}$ is positive type on $E_{n}$ if $\left[f\left(s^{-1} t\right)\right]_{s, t \in E_{n}}$ is positive semidefinite in $\mathbb{M}_{E_{n}}(\mathbb{C})$. Then by compactness, one has

$$
\begin{aligned}
\mathcal{Q}_{c} & =\bigcap_{n}\left\{\left[\varphi_{f}\left(p_{i}^{(k)} q_{j}^{(l)}\right)\right]_{k, l}: f \text { positive type on } E_{n}\right\} \\
& =\bigcap_{n}\left\{\left[\frac{1}{m^{2}} \sum_{v, w=1}^{m} \omega_{m}^{-i v-j w} f\left(s_{k}^{v} t_{l}^{w}\right)\right]_{\substack{k, l \\
i, j}}: f \text { positive type on } E_{n}\right\} .
\end{aligned}
$$

See $[\mathrm{Fr}]$ for more information. Next, we deal with $\mathcal{Q}_{s}$. Although the description

$$
\mathcal{Q}_{s}=\operatorname{closure}\left(\bigcup_{n}\left\{\left[\left\langle\left(P_{i}^{(k)} \otimes Q_{j}^{(l)}\right) \xi, \xi\right\rangle\right]_{k, l}: \begin{array}{c}
\xi \in \ell_{2}^{n} \otimes \ell_{2}^{n} \text { a unit vector, } \\
\left(P_{i}^{(k)}\right)_{i=1}^{m} \text { PVMs on } \ell_{2}^{n}, \quad \\
\left(Q_{j}^{(l)}\right)_{j=1}^{m} \text { PVMs on } \ell_{2}^{n}
\end{array}\right\}\right)
$$

is already quite good, we will do some calculations around operator system tensor products. See $[\mathrm{F}+]$ (and also $[\mathrm{FP}, \mathrm{Ka}, \mathrm{K}+]$ ) for more information on this subject. Let

$$
\mathcal{S}_{m, d}=\operatorname{span}\left\{p_{i}^{(k)}: i=1, \ldots, m, k=1, \ldots, d\right\}=\ell_{\infty}^{m}+\cdots+\ell_{\infty}^{m} \subset \mathfrak{F}_{m}^{d}
$$

be the finite-dimensional operator system. Then, $\mathcal{Q}_{s}$ coincides with the space of states on $\mathcal{S}_{m, d} \otimes^{\min } \mathcal{S}_{m, d}$, evaluated at the "basis" $\left\{p_{i}^{(k)} \otimes q_{j}^{(l)}\right\}$. By Lemma 22, the operator system structure of $\mathcal{S}_{m, d}$ is defined as follows: $a \in \mathbb{M}_{n}\left(\mathcal{S}_{m, d}\right)$ is positive if and only if there are $a_{1}, \ldots, a_{d} \in \mathbb{M}_{n}\left(\ell_{\infty}^{m}\right)_{+}$such that $a=a_{1}+\cdots+a_{m}$. This implies that a map $\varphi: \mathcal{S}_{m, d} \rightarrow \mathbb{M}_{n}(\mathbb{C})$ is c.p. if and only if the restriction of $\varphi$ to each summand $\ell_{\infty}^{m}$ is c.p. Meanwhile, a map $\psi: \ell_{\infty}^{m} \rightarrow \mathbb{M}_{n}(\mathbb{C})$ is c.p. if and only if $\psi\left(p_{i}\right) \geq 0$ for every $i=1, \ldots, m$. It follows that the dual operator system $\mathcal{S}_{m, d}^{\mathrm{d}}$ of $\mathcal{S}_{m, d}$ is naturally identified with

$$
\mathcal{S}_{m, d}^{\mathrm{d}}=\left\{\left(f_{1}, \ldots, f_{d}\right) \in \ell_{\infty}^{m} \oplus \cdots \oplus \ell_{\infty}^{m}: \sum_{i} f_{k}(i)=\sum_{j} f_{l}(j)\right\} \subset \ell_{\infty}^{d m}
$$

By Theorem 35, one has $\left(\mathcal{S}_{m, d} \otimes^{\min } \mathcal{S}_{m, d}\right)^{\mathrm{d}}=\mathcal{S}_{m, d}^{\mathrm{d}} \otimes^{\max } \mathcal{S}_{m, d}^{\mathrm{d}}$. Hence, if $c \in\left(\mathcal{S}_{m, d} \otimes^{\min } \mathcal{S}_{m, d}\right)^{\mathrm{d}}$ is strictly positive, i.e. $c \geq \varepsilon 1$ for some $\varepsilon>0$, then it has a representation $x^{*}(s \otimes t) x$ for some $x \in \mathbb{M}_{v w, 1}(\mathbb{C}), s \in \mathbb{M}_{v}\left(\mathcal{S}_{m, d}^{\mathrm{d}}\right)_{+}$, and $t \in \mathbb{M}_{w}\left(\mathcal{S}_{m, d}^{\mathrm{d}}\right)_{+}$. Viewing $x$ as a vector $\xi$ in $\ell_{2}^{v} \otimes \ell_{2}^{w}$, one has

$$
x^{*}(s \otimes t) x=\langle(s \otimes t) \xi, \xi\rangle \in \mathcal{S}_{m, d}^{\mathrm{d}} \otimes \mathcal{S}_{m, d}^{\mathrm{d}} .
$$


Evaluating it at the basis $\left\{p_{i}^{(k)} \otimes q_{j}^{(l)}\right\}$, one obtains

$$
x^{*}(s \otimes t) x=\left[\left\langle\left(s_{i}^{(k)} \otimes t_{j}^{(l)}\right) \xi, \xi\right\rangle\right]_{\substack{k, j \\ i, j}},
$$

where $s_{i}^{(k)} \in \mathbb{M}_{v}(\mathbb{C})_{+}$and $t_{j}^{(l)} \in \mathbb{M}_{w}(\mathbb{C})_{+}$satisfy $\sum_{i} s_{i}^{(k)}=a$ (independent of $k$ ) and $\sum_{j} t_{j}^{(l)}=b$ (independent of $l$ ). Replacing $\xi$ with $\left(a^{1 / 2} \otimes b^{1 / 2}\right) \xi$, one may assume that $a=1$ and $b=1$. By dilating them, one may further assume that $\left(s_{i}^{(k)}\right)_{i=1}^{m}$ and $\left(t_{j}^{(l)}\right)_{j=1}^{m}$ are PVMs. In this way, we come back to the original definition of $\mathcal{Q}_{s}$. We note that we have proved that one can realize a "generic" element $\left[\gamma_{i, j}^{k, l}\right] \in \mathcal{Q}_{s}$ by a finite-dimensional system, namely if it is faithful on $\mathcal{S}_{m, d} \otimes^{\min } \mathcal{S}_{m, d}$. It seems unlikely that this is also the case for all $\left[\gamma_{i, j}^{k, l}\right] \in \mathcal{Q}_{s}$.

\section{REFERENCES}

[BT] M. Bakonyi and D. Timotin; Extensions of positive definite functions on free groups. J. Funct. Anal. 246 (2007), 31-49.

[Ba] A. Barvinok; A course in convexity. Graduate Studies in Mathematics, 54. American Mathematical Society, Providence, RI, 2002. $\mathrm{x}+366 \mathrm{pp}$.

[Be1] M.B. Bekka; On the full $\mathrm{C}^{*}$-algebras of arithmetic groups and the congruence subgroup problem. Forum Math. 11 (1999), 705-715.

[Be2] B. Bekka; Operator-algebraic superridigity for $\mathrm{SL}_{n}(\mathbb{Z}), n \geq 3$. Invent. Math. 169 (2007), 401-425.

[Bo] F. Boca; Completely positive maps on amalgamated product $\mathrm{C}^{*}$-algebras. Math. Scand. 72 (1993), $212-222$.

[BO] N. Brown and N. Ozawa; C*-algebras and Finite-Dimensional Approximations. Graduate Studies in Mathematics, 88. American Mathematical Society, Providence, RI, 2008.

[C+] J. Carrión, M. Dadarlat, and C. Eckhardt; On groups with quasidiagonal $\mathrm{C}^{*}$-algebras. Preprint. arXiv: 1210.4050

[CE] M.D. Choi and E.G. Effros; Injectivity and operator spaces. J. Funct. Anal. 24 (1977), 156-209.

[Ci] J. Cimprič; A representation theorem for Archimedean quadratic modules on $*$-rings. Canad. Math. Bull. 52 (2009), 39-52.

[Co] A. Connes; Classification of injective factors. Ann. of Math. (2) 104 (1976), 73-115.

[DJ] K. Dykema and K. Juschenko; Matrices of unitary moments. Math. Scand. 109 (2011), 225-239.

[EL] R. Exel and T.A. Loring; Finite-dimensional representations of free product $\mathrm{C}^{*}$-algebras. Internat. J. Math. 3 (1992), 469-476.

[FH] T. Fack and P. de la Harpe; Sommes de commutateurs dans les algèbres de von Neumann finies continues. Ann. Inst. Fourier (Grenoble) 30 (1980), 49-73.

$[\mathrm{F}+]$ D. Farenick, A.S. Kavruk, V.I. Paulsen, and I.G. Todorov; Operator systems from discrete groups. Preprint. arXiv: 1209.1152

[FP] D. Farenick and V I. Paulsen; Operator system quotients of matrix algebras and their tensor products. Math. Scand., to appear. arXiv:1101.0790

[Fr] T. Fritz; Tsirelson's problem and Kirchberg's conjecture. Rev. Math. Phys. 24 (2012), 1250012, 67 pp.

[HM] J.W. Helton and S.A. McCullough; A Positivstellensatz for non-commutative polynomials. Trans. Amer. Math. Soc. 356 (2004), 3721-3737. 
[J+] M. Junge, M. Navascues, C. Palazuelos, D. Perez-Garcia, V.B. Scholz, and R.F. Werner; Connes embedding problem and Tsirelson's problem. J. Math. Phys. 52 (2011), 012102, 12 pp.

[JP] K. Juschenko and S. Popovych; Algebraic reformulation of Connes embedding problem and the free group algebra. Israel J. Math. 181 (2011), 305-315.

[Ka] A.S. Kavruk; The weak expectation property and riesz interpolation. Preprint. arXiv:1201.5414

$[\mathrm{K}+]$ A. Kavruk, V.I. Paulsen, I.G. Todorov, and M. Tomforde; Tensor products of operator systems. J. Funct. Anal. 261 (2011), 267-299.

[Ki1] E. Kirchberg; On nonsemisplit extensions, tensor products and exactness of group $\mathrm{C}^{*}$-algebras. Invent. Math. 112 (1993), 449-489.

[Ki2] E. Kirchberg; Discrete groups with Kazhdan's property $T$ and factorization property are residually finite. Math. Ann. 299 (1994), 551-563.

[KS] I. Klep and M. Schweighofer; Connes' embedding conjecture and sums of Hermitian squares. Adv. Math. 217 (2008), 1816-1837.

[LS] A. Lubotzky and Y. Shalom; Finite representations in the unitary dual and Ramanujan groups. Discrete geometric analysis, 173-189, Contemp. Math., 347, Amer. Math. Soc., Providence, RI, 2004.

[Mc] S. McCullough; Factorization of operator-valued polynomials in several non-commuting variables. Linear Algebra Appl. 326 (2001), 193-203.

[NT] T. Netzer and A. Thom; Real closed separation theorems and applications to group algebras. Pacific J. Math., to appear. arXiv:1110.5619

[Oz1] N. Ozawa; About the QWEP conjecture. Internat. J. Math. 15 (2004), 501-530.

[Oz2] N. Ozawa; Tsirelson's Problem and Asymptotically Commuting Unitary Matrices. J. Math. Phys., accepted. arXiv:1211.2712

[Pa] V. Paulsen; Completely bounded maps and operator algebras. Cambridge Studies in Advanced Mathematics, 78. Cambridge University Press, Cambridge, 2002. xii+300 pp.

[Pi1] G. Pisier; Introduction to operator space theory. London Mathematical Society Lecture Note Series, 294. Cambridge University Press, Cambridge, 2003. viii+478 pp.

[Pi2] G. Pisier; Grothendieck's theorem, past and present. Bull. Amer. Math. Soc. (N.S.) 49 (2012), 237-323.

[Pu] M. Putinar; Positive polynomials on compact semi-algebraic sets. Indiana Univ. Math. J. 42 (1993), 969-984.

[Ru $\quad$ W. Rudin; The extension problem for positive-definite functions. Illinois J. Math. 7 (1963), 532 539.

[Sd] C. Scheiderer; Sums of squares on real algebraic surfaces. Manuscripta Math. 119 (2006), 395-410.

[Sm] K. Schmüdgen; Noncommutative real algebraic geometry - some basic concepts and first ideas. Emerging applications of algebraic geometry, 325-350, IMA Vol. Math. Appl., 149, Springer, New York, 2009.

[Ta] M. Takesaki; Theory of operator algebras II. Encyclopedia of Mathematical Sciences, 125. Operator Algebras and Non-commutative Geometry, 5. Springer-Verlag, Berlin, 2002.

[Ts] B.S. Tsirelson; Some results and problems on quantum Bell-type inequalities. Fundamental questions in quantum physics and relativity, 32-48, Hadronic Press Collect. Orig. Artic., Hadronic Press, Palm Harbor, FL, 1993.

RIMS, KYOTO UNIVERSITY, 606-8502

E-mail address: narutaka@kurims.kyoto-u.ac.jp 